\title{
Random Berry Phase Magnetoresistance as a Probe of Interface Roughness in Si MOSFETs
}

\author{
H. Mathur ${ }^{1}$ and Harold U. Baranger ${ }^{2}$ \\ ${ }^{1}$ Department of Physics, Case Western Reserve University, Cleveland OH 44106-7079 \\ ${ }^{2}$ Department of Physics, Duke University, Box 90305, Durham NC 27708-0305
}

(October 30, 2018)

\begin{abstract}
The effect of silicon-oxide interface roughness on the weaklocalization magnetoconductance of a silicon MOSFET in a magnetic field, tilted with respect to the interface, is studied. It is shown that an electron picks up a random Berry's phase as it traverses a closed orbit. Effectively, due to roughness, the electron sees an uniform field parallel to the interface as a random perpendicular field. At zero parallel field the dependence of the conductance on the perpendicular field has a well known form, the weak-localization lineshape. Here the effect of applying a fixed parallel field on the lineshape is analyzed. Many types of behavior are found including homogeneous broadening, inhomogeneous broadening and a remarkable regime in which the change in lineshape depends only on the magnetic field, the two length scales that characterize the interface roughness and fundamental constants. Good agreement is obtained with experiments that are in the homogeneous broadening limit. The implications for using weak-localization magnetoconductance as a probe of interface roughness, as proposed by Wheeler and coworkers, are discussed.
\end{abstract}

PACS:

\section{INTRODUCTION}

Disorder has a profound effect on electron transport at low temperature. The scaling theory of localization applies over an enormous domain and is a keystone in our understanding of disorder effects [1,2]. For twodimensional samples that are weakly-disordered and at low temperature ("weak-localization regime") the theory predicts the precise dependence of the conductance on an applied magnetic field ("weak-localization lineshape") [3]. The exquisite agreement of the predicted lineshape with experiment constitutes an important confirmation of scaling theory [4].

Silicon MOSFETs are an important experimental realization of a two-dimensional electronic system. In a MOSFET electrons are confined to the interface between layers of oxide and semiconductor. In this paper we analyze the effect of interface roughness on the weaklocalization lineshape in MOSFETs. Although the effects are small they are of interest from various points of view:

i) The deviations from the known lineshape of an ideal interface are small but measurable: our results agree well with the experiments that stimulated our work [5]. ii) The relevant effect of roughness on the electrons can be traced to a subtle quantum interference effect: Berry's phase [8.97. There has been considerable theoretical interest in designing an experiment sensitive to the influence of Berry's phase on quantum electron transport [10]. Here we have identified a prior experimental detection of it.

iii) A remarkable feature of many quantum transport phenomena is their universality, in the sense that the observed effects are independent of microscopic sample properties such as the mean mobility. The effects of roughness have this feature. We find a particularly striking regime in which the effect is determined entirely by the geometric parameters of the interface and the applied magnetic field while being independent of both sample mobility and temperature (which controls the dephasing length).

iv) There has been much experimental and theoretical work on the motion of electrons in a random magnetic field, particularly in the strong field limit [11 17] (motivated in part by possible relevance to cuprates and the quantum Hall system at filling factor $1 / 2$ ). We find that due to roughness electrons see a uniform in-plane magnetic field as a random perpendicular field; hence it may be possible to use this system to study electron motion in a random magnetic field. Here our analysis is restricted to the weak field limit appropriate for calculating the lineshape in MOSFETs. Experiments on other realizations of the random field problem are briefly discussed in Section VI.

v) Finally this work may have practical implications. MOSFETs are the building blocks of modern electronics. Roughness of the interface between the oxide and semiconductor influences the mobility of the device and has been correlated with device failure due to dielectric breakdown of the oxide 18,19. It is therefore of technological interest to characterize the roughness. R. G. Wheeler and co-workers have proposed that magnetoresistance measurements on a MOSFET in the weaklocalization regime can be used as a non-destructive probe of its interface roughness [5.6]. They and others have carried out experiments that demonstrate the feasibility of making the needed measurements 5 6]. The present work contributes to this program by providing the precise relationship between the interface roughness parameters and the measured magnetoresistance.

Figs. 1 and 2 summarize our findings. Following the 


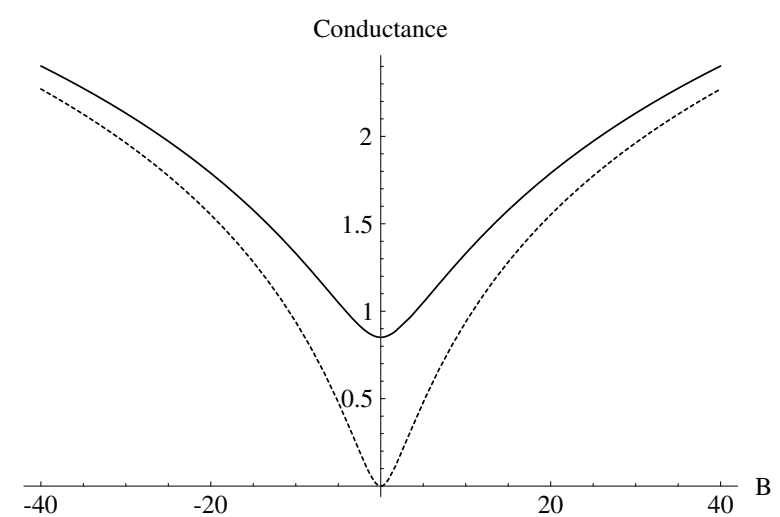

FIG. 1. MOSFET with short-ranged correlated roughness $\left(L \ll l_{e}\right)$. The conductance (in units of $10^{-5} \mathrm{mhos}$ ) is plotted as a function of the applied perpendicular field, $B_{\perp} / B_{\phi}$. The dotted curve is the classic weak-localization lineshape. It corresponds to zero in-plane field, $B_{\|}=0$. The solid curve shows the effect of applying a fixed in-plane magnetic field. It corresponds to $B_{e} / B_{\phi}=400$ and $B_{\|} / B_{\phi}=\left(\sqrt{8} / \pi^{1 / 4}\right) \sqrt{l_{e} l_{\phi}^{2} / L \Delta^{2}}$.

experiments of Ref. [5.6] we imagine the device is placed in a magnetic field tilted with respect to the plane of the interface. The in-plane component of the field, $B_{\|}$is kept fixed and the conductance is plotted as a function of the perpendicular component, $B_{\perp}$. To understand these curves it is useful to recall that there are two important length scales that determine the transport properties of the sample: $l_{e}$, the elastic mean free path of electrons (which determines the mobility), and $l_{\phi}$, the distance over which electrons maintain phase-coherence and are thus able to interfere. In the weak-localization regime, $l_{\phi} \gg l_{e}$. Also, atomic force microscope images reveal that statistically the rough interface can be characterized by two parameters: $\Delta=$ the root-mean-square height fluctuations and $L=$ the distance over which the fluctuations are correlated.

For $B_{\|}=0$ we obtain the classic weak-localization lineshape appropriate for an ideal interface (dotted curves in Figs. 1 and 2). The height 1 and the width of the peak are controlled by the dephasing length $l_{\phi}$. The solid curves show possible lineshapes when the parallel field is turned on. Fig. 1 shows that when the roughness is correlated over a very short length scale $\left(L \ll l_{e}\right)$ the effect of the parallel field is to decrease the dephasing length; the lineshape is otherwise unaltered. Borrowing the terminology of magnetic resonance and atomic physics the effect of the in-plane field in this limit may be described as homogeneous line-broadening. In the opposite limit when

\footnotetext{
${ }^{1}$ The height is measured relative to the Drude conductance given by the large-field asymptotic value of the conductance corrected for classical magnetoresistance.
}

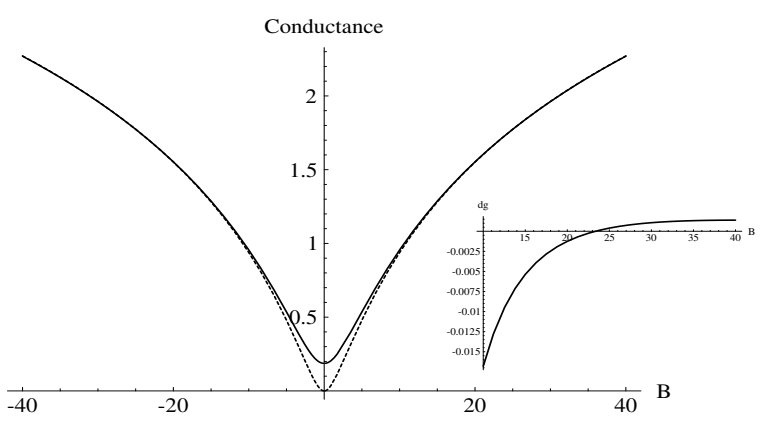

FIG. 2. Same as Fig. 1 for a MOSFET with long-range correlated roughness $\left(L \gg l_{e}\right)$. The dotted curve corresponds to $B_{\|}=0$; the solid curve, to $B_{\|} \Delta L=\phi_{\mathrm{sc}} / 10$. Also $B_{e}=400 B_{\phi}$ and $L=l_{\phi} / \sqrt{5}$. The crossing of the two curves is highlighted by a plot of their difference (inset).

the interface fluctuates slowly $\left(L \gg l_{e}\right)$, the effects are more dramatic. The deviation from the ideal lineshape changes sign as a function of perpendicular field (Fig. 2 , inset). Again, by analogy to magnetic resonance and atomic physics, the extreme limit, $L \gg l_{\phi} \gg l_{e}$, can be interpreted as inhomogeneous broadening (see Section $\mathrm{V}$ ). The sign change is then traceable to a necessary inflection point in the ideal weak-localization lineshape. The intermediate regime $l_{\phi} \gg L \gg l_{e}$ is particularly interesting although it cannot be so simply interpreted. In this limit the deviation from the ideal lineshape has a universal form independent of $l_{e}$ and $l_{\phi}$. For example, the sign change occurs at a value of the perpendicular field determined by the purely geometric condition $B_{\perp} L^{2}=(1.79 \ldots) h / e$. Note that in all cases the deviation from the ideal lineshape grows with $B_{\|}$and $\Delta$ (in proportion to $B_{\|}^{2}$ and $\Delta^{2}$ in the experimentally relevant regime).

Further discussion and a detailed summary of our results is given in Section VII.

\section{BORN-OPPENHEIMER ANALYSIS}

As a model of the silicon-oxide interface, for many purposes it is sufficient to regard the oxide as an impenetrable hard wall and to assume that the electrons are bound to the interface by a uniform perpendicular electric field [20]. It will become apparent in the sequel that for the present purpose it is only necessary to assume that the electrons are firmly bound to the interface: it is not necessary to commit to a specific form of the confinement potential $U_{\text {conf }}$. If the axes are chosen so that the interface lies in the plane $z=0$, for an ideal interface the confinement potential would depend only on $z$; but for a rough interface it would vary from point to point and hence also depend on $x$ and $y$. The strength of the confining potential controls the extent of the wavefunction in the $z$ 
direction, denoted $\ell$. We also allow for the possibility of electron scattering by impurities in the semiconductor by introducing a potential $U_{\text {imp. }}$. The magnetic field is assumed to lie in the $x-z$ plane: thus $B_{x}=B_{\|}, B_{y}=0$ and $B_{z}=B_{\perp}$. It is convenient to work in a Landau gauge and to choose $A_{x}=0, A_{y}=B_{\perp} x$ and $A_{z}=B_{\|} y$. The Schrödinger equation is

$$
\begin{aligned}
& -\frac{\hbar^{2}}{2 m}\left(\frac{\partial}{\partial z}-i e \frac{A_{z}}{\hbar}\right)^{2} \Psi-\frac{\hbar^{2}}{2 m}\left(\nabla-i e \frac{\mathbf{A}}{\hbar}\right)^{2} \Psi \\
& +U_{\operatorname{conf}}(z, x, y) \Psi+U_{\mathrm{imp}}(z, x, y) \Psi=E \Psi(x, y, z) .
\end{aligned}
$$

For later convenience $\nabla$ is used to denote the twodimensional gradient in the $x-y$ plane. Similarly A denotes the $x-y$ component of the vector potential.

For an ideal interface the electronic motion in the plane of the interface and in the perpendicular direction separate. The motion in the perpendicular $z$-direction is quantized by the confining potential. Provided the temperature is sufficiently low and the density of electrons not too high, only the lowest subband mode in the $z$ direction is populated and the electronic motion is essentially two-dimensional. For a rough interface the motion no longer separates but we find it is an excellent approximation to integrate out the motion perpendicular to the interface and to obtain an effective Hamiltonian for motion in the plane of the interface using the BornOppenheimer method 21.

For simplicity first suppose there is no magnetic field. Assume that the motion in the $x-y$ plane is slow; the motion perpendicular to it, fast. The first step in the Born-Oppenheimer method is to analyze the motion of the fast co-ordinates treating the slow co-ordinates as parameters. In this case it is necessary to solve

$$
\begin{array}{r}
-\frac{\hbar^{2}}{2 m} \frac{d^{2}}{d z^{2}} \phi_{n}(z ; x, y)+U_{\operatorname{conf}}(z, x, y) \phi_{n}(z ; x, y) \\
=E_{n}(x, y) \phi_{n}(z ; x, y)
\end{array}
$$

Here $\phi_{n}(z ; x, y)$ denotes the "local subband" wavefunction and $E_{n}(x, y)$ is the energy of the $n^{\text {th }}$ subband wavefunction. Sometimes it will be convenient to write the sub-band states using Dirac notation: $\phi_{n}(z ; x, y) \rightarrow$ $|n ; x, y\rangle$. Both the subband wavefunction and the subband energy vary with location in the $x-y$ plane because the confinement potential varies from point to point. It is assumed that the subband wavefunction is normalized everywhere so that $\langle n ; x, y \mid n ; x, y\rangle=\int_{-\infty}^{\infty} d z\left|\phi_{n}(z ; x, y)\right|^{2}=$ 1. For a given $(x, y)$ Eq. (2) only fixes the subband wavefunction up to a phase. It is convenient to choose the wavefunctions to be real and to vary smoothly with $x$ and $y$.

According to the Born-Oppenheimer method an approximate solution to Eq. (1) is

$$
\Psi(x, y, z) \approx \psi(x, y) \phi_{g}(z ; x, y)
$$

Here $\phi_{g}$ denotes the lowest energy subband wavefunction and $\psi(x, y)$ is governed by

$$
\begin{aligned}
& -\frac{\hbar^{2}}{2 m} \nabla^{2} \psi(x, y)+\tilde{U}_{\mathrm{imp}}(x, y) \psi(x, y) \\
& +E_{g}(x, y) \psi(x, y)+W_{g}(x, y) \psi(x, y)=E \psi(x, y) .
\end{aligned}
$$

Eq. (4) describes the motion of the electrons in the plane of the interface after the transverse motion has been integrated out. $\tilde{U}_{\text {imp }}$, the effect of impurities, now depends only on $x$ and $y$. For impurities that are sufficiently far from the interface so that their potential does not vary significantly over the confinement scale $l, \tilde{U}_{\mathrm{imp}}=U_{\mathrm{imp}}$, while for short-range impurities $\tilde{U}_{\text {imp }}$ incorporates their effect on electrons in the lowest subband. Note that the local subband energy, $E_{g}(x, y)$ appears as a potential in the Born-Oppenheimer effective Hamiltonian. The effective potential $W_{g}(x, y)$ is also determined by the solution to the fast problem. It is given by a more complicated expression

$$
W_{g}(x, y)=\sum_{n \neq g}\langle g ; x, y|(\nabla|n ; x, y\rangle) \cdot\langle n ; x, y|\nabla| g ; x, y\rangle .
$$

Next suppose that the magnetic field is turned on. The fast co-ordinate is now governed by

$$
\begin{aligned}
& -\frac{\hbar^{2}}{2 m}\left(\frac{d}{d z}-i \frac{e}{\hbar} B_{\|} y\right)^{2} \phi_{n}(z ; x, y) \\
& +U_{\operatorname{conf}}(z, x, y) \phi_{n}(z ; x, y)=E_{n}(x, y) \phi_{n}(z ; x, y) .
\end{aligned}
$$

Let $\xi_{n}(z ; x, y)$ denote a normalized solution to Eq. (6) when the magnetic field is turned off. This solution is chosen to be real and to vary smoothly with $x$ and $y$. It is easy to verify that a solution to Eq. (6) with the magnetic field is

$$
\phi_{n}(z ; x, y)=\exp \left(i \frac{e}{\hbar} B_{\|} y z\right) \xi_{n}(z ; x, y) .
$$

Turning on the magnetic field leaves the subband energy, $E_{n}(x, y)$ unchanged; but it introduces a non-trivial twist in the subband wavefunctions due to the phase factor in Eq. (7). Following Berry [8,9], this can be made explicit by defining the geometric vector potential $\mathbf{A}_{g}(x, y) \equiv i(\hbar / e)\langle g ; x, y|\nabla| g ; x, y\rangle$ and its curl, the geometric magnetic field. Straightforward calculation reveals that the cartesian components of $\mathbf{A}$ are

$$
\begin{aligned}
& A_{g x}(x, y)=0 \\
& A_{g y}(x, y)=-B_{\|} \mathcal{Z}_{g}(x, y)
\end{aligned}
$$

and the geometric magnetic field is

$$
\begin{aligned}
B_{g}(x, y) & \equiv \frac{\partial}{\partial x} A_{g y}-\frac{\partial}{\partial y} A_{g x} \\
& =-B_{\|} \frac{\partial}{\partial x} \mathcal{Z}_{g}(x, y)
\end{aligned}
$$


Here $\mathcal{Z}_{g}(x, y)$ is the mean $z$-coordinate for the lowest subband wavefunction,

$$
\mathcal{Z}_{g}(x, y) \equiv \int_{-\infty}^{\infty} d z z\left|\xi_{g}(z ; x, y)\right|^{2} .
$$

Now, according to the Born-Oppenheimer method, an approximate solution to Eq. (1) is given by Eq. (3). Here $\phi_{g}$ denotes the lowest energy subband wavefunction and $\psi(x, y)$ is governed by

$$
\begin{aligned}
& -\frac{\hbar^{2}}{2 m}\left(\nabla-i \frac{e}{\hbar}\left[\mathbf{A}(x, y)+\mathbf{A}_{g}(x, y)\right]\right)^{2} \psi(x, y) \\
& +E_{g}(x, y) \psi(x, y)+W_{g}(x, y) \psi(x, y) \\
& +\tilde{U}_{\mathrm{imp}}(x, y) \psi(x, y)=E \psi(x, y) .
\end{aligned}
$$

Eq. (11) is the central result of this section. It reveals that after the fast motion perpendicular to the interface is integrated out, the electrons essentially move in two dimensions under the influence of an effective potential, $\tilde{U}_{\text {imp }}+E_{g}+W_{g}$ and an effective perpendicular magnetic field, $B_{\perp}+B_{g}$.

The effective magnetic field is seen to be the component of the applied field that is perpendicular to the surface defined by the mean $z$-coordinate of the subband wavefunctions, $\mathcal{Z}_{g}(x, y)$ provided that the gradients in this surface are small. Note that the geometric magnetic field, $B_{g}$ given by Eq. (9) is proportional to the in-plane component of the applied field and to the gradient of the surface $\mathcal{Z}_{g}$. It vanishes if $B_{\|}=0$ or for an ideal interface for which the surface $\mathcal{Z}_{g}$ would be flat. The effective potentials $E_{g}$ and $W_{g}$ also owe their existence to the roughness of the interface but are independent of $B_{\|}$.

Note that Eq. (6) only defines the subband wavefunctions, $\phi_{n}$ up to a phase. A specific choice given by Eq. (7) is made in the calculation above. This amounts to choosing a gauge for the geometric vector potential: a different choice of phase would transform the geometric vector potential, $\mathbf{A}_{g}$, but would leave the geometric magnetic field, $B_{g}$ unchanged. Thus the calculation above uses a specific gauge for both the applied and geometric magnetic field - the Landau gauge defined before Eq. (1) and the gauge given by Eq. (8), respectively. The gauges are chosen for their convenience but the results are, of course, independent of the choice of gauge. A fuller discussion of the gauge invariance of the Born-Oppenheimer method is given in chapter 3.7 of Ref. [9].

Finally we briefly discuss the applicability of the BornOppenheimer approximation. In Appendix A it is shown that the approximation should work provided $\ell \ll \lambda_{f}$, $\nabla \mathcal{Z}_{g} \ll 1$ and $\ell \nabla^{2} \mathcal{Z}_{g} \ll 1$. Here $\ell$ is the typical extent of the subband wavefunction in the $z$-direction and $\lambda_{f}$ is the Fermi wavelength of the electrons. The first condition is to ensure that the electron density is not so high that more than one subband is occupied. The other two inequalities are adiabaticity requirements on the surface roughness needed to ensure that the motion in the plane of the interface can be approximately decoupled from the transverse motion. They stipulate that the roughness must vary slowly on a scale determined by the strength of the confining potential.

To check the validity of the adiabaticity conditions we can use the atomic force microscope images of the siliconoxide interface presented in Ref. [6]. These images reveal that the height fluctuations of the interface follow a Gaussian distribution parameterized by $\Delta_{\mathrm{AFM}}$, the root mean square height fluctuation, and $L$, the correlation distance. The discussion of Appendix A suggests that the surface $\mathcal{Z}_{g}$ should be similarly distributed with essentially the same parameters. Hence the adiabaticity conditions can be expressed in terms of the observable parameters as $\Delta_{\mathrm{AFM}} / L \ll 1$ and $\left(\ell \Delta_{\mathrm{AFM}}\right) / L^{2} \ll 1$ and are seen to be very well satisfied for both samples of Ref. [6].

\section{WEAK-LOCALIZATION LINESHAPE}

The purpose of this section is to review the calculation of the weak localization lineshape. The calculation is reformulated in a way that is suitable for the eventual goal of calculating the tilted field magnetoresistance of a MOSFET with a rough interface.

In the previous section it was shown that effectively the electrons move in two dimensions under the influence of a random potential, $V(x, y)$, and a perpendicular magnetic field. The magnetic field is the sum of the applied perpendicular field and the geometric field, which is due to interface roughness and the applied parallel field. It is assumed that the surface $\mathcal{Z}_{g}(x, y)$ is a Gaussian random surface with zero mean and variance given by

$$
\left\langle\mathcal{Z}_{g}(\mathbf{r}) \mathcal{Z}_{g}\left(\mathbf{r}^{\prime}\right)\right\rangle_{\text {rough }}=\Delta^{2} \exp \left(-\frac{\left|\mathbf{r}-\mathbf{r}^{\prime}\right|^{2}}{L^{2}}\right) .
$$

As noted above, atomic force microscope images of the silicon-oxide interface presented in Ref. [6] reveal a Gaussian random surface, which makes it extremely plausible that the surface $\mathcal{Z}_{g}(x, y)$ must also be Gaussian. An argument to this effect is given in Appendix A. Eqs. (9) and (12) thus determine the statistics of the random magnetic field, $B_{g}$.

The random potential $V$ is caused by impurities and by the roughness of the interface (in the notation of the previous section $\left.V=U_{\mathrm{imp}}+E_{g}+W_{g}\right)$. Here we shall assume that the random potential is Gaussian white noise with zero mean and variance $\left\langle V(\mathbf{r}) V\left(\mathbf{r}^{\prime}\right)\right\rangle_{\text {imp }}=V_{0}^{2} \delta^{(2)}\left(\mathbf{r}-\mathbf{r}^{\prime}\right)$. Since we assume that the interface roughness is correlated over a length scale $L$, strictly the assumption that the random potential is Gaussian white noise is justified only under special circumstances (if the impurity potential is white noise and it dominates interface roughness scattering, or if $L$ is much smaller than all relevant length 
scales and the impurity scattering is either also white noise or is dominated by interface roughness scattering), but in practice it is reasonable to believe that our results will be more broadly applicable since localization effects are believed to be insensitive to microscopic details of the random potential. We estimate that for both samples studied in Ref. [6] impurity scattering dominates interface roughness scattering, and for one sample $L$ is shorter than all relevant length scales in addition; but it is expected that the results should apply under less favorable circumstances also.

It is useful to consider the electron Green function which obeys the Schrödinger equation

$$
\begin{aligned}
& {\left[-\frac{\hbar^{2}}{2 m}\left(\nabla-i \frac{e}{\hbar}\left[\mathbf{A}+\mathbf{A}_{g}\right]\right)^{2}+V(x, y)-E\right] \mathcal{G}\left(\mathbf{r}, \mathbf{r}^{\prime} ; E\right)} \\
& =-\delta^{(2)}\left(\mathbf{r}-\mathbf{r}^{\prime}\right) .
\end{aligned}
$$

For the retarded Green function $\mathcal{G}^{R}$, the energy $E$ has a positive infinitesimal imaginary part; for the advanced, $\mathcal{G}^{A}$, a negative part. Linear response theory allows us to express the conductance in terms of the Green functions 22

$g=-\frac{e^{2} \hbar^{3}}{8 \pi m^{2} L^{2}} \int d \mathbf{r} \int d \mathbf{r}^{\prime} \Delta \mathcal{G}\left(\mathbf{r}, \mathbf{r}^{\prime}, E_{f}\right) \frac{\stackrel{\leftrightarrow}{\partial}}{\partial y} \frac{\overleftrightarrow{\partial}}{\partial y^{\prime}} \Delta \mathcal{G}\left(\mathbf{r}^{\prime}, \mathbf{r}, E_{f}\right)$

Here $\Delta \mathcal{G} \equiv \mathcal{G}^{R}-\mathcal{G}^{A}, E_{F}=$ Fermi energy and $m=$ effective mass of electrons. We have assumed that the sample is rectangular (dimensions $L \times W$ ) and current is driven along the length of the sample which is oriented along the $y$-axis. The two-sided derivative in Eq. (14) is defined as

$$
f \frac{\stackrel{\leftrightarrow}{\partial}}{\partial y} g \equiv f \frac{\partial}{\partial y} g-g \frac{\partial}{\partial y} f
$$

We are interested in the disorder averaged conductance. To this end it is necessary to average products of Green functions over the random potential and the interface roughness under the statistical assumptions made above.

In the two subsections below we review the calculation of the conductance at zero magnetic field and in a uniform perpendicular field, circumstances under which there is no random magnetic field and it is only necessary to perform an average over the random potential. The effect of the random magnetic field is analyzed in the following sections.

\section{A. Zero Field}

The average over the random potential can be calculated perturbatively 22,23] in an expansion in the small parameter $\left(E_{f} \tau_{e}\right)^{-1}$. Here $\tau_{e}$, the elastic scattering time

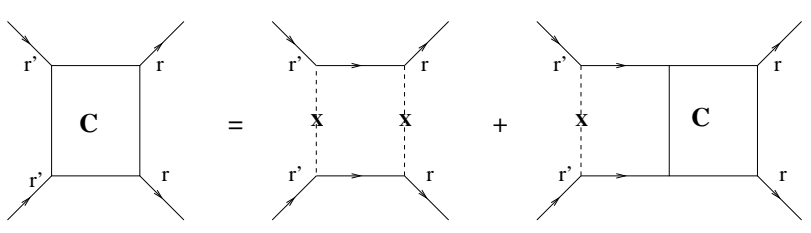

FIG. 3. Cooperon diagrams. Solid lines denote impurity averaged Green functions. The upper lines are retarded Green functions; the lower lines, advanced. Dotted lines represent scattering from impurities (shown as crosses).

for electrons, is given by $\hbar / 2 \pi \rho\left(E_{f}\right) V_{0}^{2}$. where $\rho\left(E_{f}\right)=$ density of states for spinless electrons. In this approximation the average Green function is given by 223.

$$
\begin{aligned}
G^{R}\left(\mathbf{r}, \mathbf{r}^{\prime}, E_{f}\right) & =\int \frac{d \mathbf{k}}{(2 \pi)^{2}} G^{R}\left(\mathbf{k}, E_{f}\right) \exp i \mathbf{k} \cdot\left(\mathbf{r}-\mathbf{r}^{\prime}\right) \\
G^{R}\left(\mathbf{k}, E_{f}\right) & =\left(E_{f}-\frac{\hbar^{2} k^{2}}{2 m}+i \frac{\hbar}{2 \tau_{e}}\right)^{-1}
\end{aligned}
$$

Here $G\left(\mathbf{r}, \mathbf{r}^{\prime} ; E_{f}\right)=\left\langle\mathcal{G}\left(\mathbf{r}, \mathbf{r}^{\prime} ; E_{f}\right)\right\rangle_{\text {imp }}$ is the Green function averaged over the random potential.

The average of a product of Green functions does not factorize into a product of the averages; it is correlated by the underlying random potential. The correlation between $\mathcal{G}^{R}$ and $\mathcal{G}^{A}$ responsible for weak localization is called the Cooperon, $C\left(\mathbf{r}, \mathbf{r}^{\prime}\right)$. Semiclassically this correlation can be understood to arise from the constructive interference of classical paths related by time reversal symmetry 目田. Fig. 3 shows the Feynman diagrams that contribute to the Cooperon [1]. From these diagrams we see that it obeys the integral equation

$$
C\left(\mathbf{r}, \mathbf{r}^{\prime}\right)=C^{(0)}\left(\mathbf{r}, \mathbf{r}^{\prime}\right)+\int d \mathbf{r}^{\prime \prime} C^{(0)}\left(\mathbf{r}, \mathbf{r}^{\prime \prime}\right) C\left(\mathbf{r}^{\prime \prime}, \mathbf{r}^{\prime}\right) .
$$

Here $C^{(0)}\left(\mathbf{r}, \mathbf{r}^{\prime}\right)=\left(\hbar / 2 \pi \rho\left(E_{f}\right) \tau_{e}\right)\left|\left\langle G^{R}\left(\mathbf{r}, \mathbf{r}^{\prime}, E_{f}\right)\right\rangle_{\text {imp }}\right|^{2}$. Using Eqs. (14) and (16), the weak-localization contribution to the conductance, $g_{\mathrm{WL}}$, can be expressed in terms of the full Cooperon:

$$
g_{\mathrm{WL}}=-\frac{2}{\pi} \frac{e^{2}}{L^{2}} D \tau_{e} \int d \mathbf{r} C(\mathbf{r}, \mathbf{r})
$$

Here $D \equiv v_{f}^{2} \tau_{e} / 2$ is the electron diffusion constant. Calculation of $g_{\mathrm{WL}}$ therefore reduces to solution of Eq. (17).

The customary procedure is to obtain eigenfunctions of $C^{(0)}$ which obey

$$
\int d \mathbf{r}^{\prime} C^{(0)}\left(\mathbf{r}, \mathbf{r}^{\prime}\right) Q_{\lambda}\left(\mathbf{r}^{\prime}\right)=\lambda Q_{\lambda}(\mathbf{r})
$$

The solution to Eq. (17) is then

$$
C\left(\mathbf{r}, \mathbf{r}^{\prime}\right)=\sum_{\lambda}\left(\frac{\lambda}{1-\lambda}\right) Q_{\lambda}(\mathbf{r}) Q_{\lambda}\left(\mathbf{r}^{\prime}\right) .
$$


It turns out that eigenfunctions that vary slowly on the scale of $l_{e}=v_{f} \tau_{e}$ dominate the conductance; hence, it is only necessary to accurately calculate the slowly varying solutions of Eq. (19). Asymptotic evaluation of the Green function, Eq. (16), shows that

$$
C^{(0)}\left(\mathbf{r}, \mathbf{r}^{\prime}\right) \approx \frac{1}{2 \pi l_{e}^{2}} \exp -\frac{\left|\mathbf{r}-\mathbf{r}^{\prime}\right|}{l_{e}}
$$

provided $\left|\mathbf{r}-\mathbf{r}^{\prime}\right| \gg \lambda_{f}$ and $k_{f} l_{e} \gg 1 . C^{(0)}$ is therefore sharply peaked about $\mathbf{r} \approx \mathbf{r}^{\prime}$ and for the slowly varying eigenfunctions the integral in Eq. (19) can be performed approximately by expanding $Q_{\lambda}\left(\mathbf{r}^{\prime}\right)$ in a Taylor's series about $\mathbf{r}^{\prime}=\mathbf{r}$. Keeping terms to second order transforms the integral equation (19) into a diffusion equation

$$
D \tau_{e} \nabla^{2} Q_{\lambda}(\mathbf{r})+Q_{\lambda}(\mathbf{r})=\lambda Q_{\lambda}(\mathbf{r}) .
$$

It is sufficient to use the solutions of the diffusion Eq. (22) to construct the Cooperon, Eq. (20), and to calculate the conductance using Eq. (18). This completes the usual calculation at zero field.

We now introduce an alternative formulation more suitable for analyzing the effects of roughness. Consider the kernel

$$
K^{(0)}\left(\mathbf{r}, \mathbf{r}^{\prime}\right) \equiv \frac{1}{2 \pi l_{e}^{2}} \exp \left(-\frac{\left|\mathbf{r}-\mathbf{r}^{\prime}\right|^{2}}{2 l_{e}^{2}}\right) .
$$

By analogy with $C^{(0)}$ and $C$ introduce $K$ given by

$$
K\left(\mathbf{r}, \mathbf{r}^{\prime}\right)=K^{(0)}\left(\mathbf{r}, \mathbf{r}^{\prime}\right)+\int d \mathbf{r}^{\prime \prime} K\left(\mathbf{r}, \mathbf{r}^{\prime \prime}\right) K^{(0)}\left(\mathbf{r}^{\prime \prime}, \mathbf{r}^{\prime}\right) .
$$

Note that $K^{(0)}$ is not equal to $C^{(0)}$ [compare Eqs. (21) and (23)]; but the slowly varying eigenfunctions of $K^{(0)}$ also obey the diffusion Eq. (22). For this reason $K$ and $C$ have the same long distance behavior. Thus, we may use $K$ instead of $C$ in Eq. (18) to calculate the weaklocalization conductance since it is dominated by the longdistance behavior.

The chief virtue of $K$ compared to $C$ is that it can be expressed in terms of tractable Gaussian integrals. A useful expression for $K$ is obtained from Eq. (24) by iteration

$$
\begin{aligned}
K\left(\mathbf{r}, \mathbf{r}^{\prime}\right)= & \sum_{n=0}^{\infty} K^{(n)}\left(\mathbf{r}, \mathbf{r}^{\prime}\right) \\
K^{(n)}\left(\mathbf{r}, \mathbf{r}^{\prime}\right)= & \int d \mathbf{r}_{1} \ldots d \mathbf{r}_{n} K^{(0)}\left(\mathbf{r}, \mathbf{r}_{1}\right) K^{(0)}\left(\mathbf{r}_{1}, \mathbf{r}_{2}\right) \ldots \\
& \ldots K^{(0)}\left(\mathbf{r}_{n}, \mathbf{r}^{\prime}\right) .
\end{aligned}
$$

Evidently $K^{(n)}\left(\mathbf{r}, \mathbf{r}^{\prime}\right)$ may be interpreted as the probability for a Gaussian random walker to go from $\mathbf{r}^{\prime}$ to $\mathbf{r}$ in $n+1$ steps.

An important physical ingredient must now be added. As noted above, weak-localization is a quantum interference effect. It is therefore damped by electron-electron and electron-phonon scattering [24]. In the conventional analysis the damping effect of these processes is included phenomenologically by adding $\tau_{e} / \tau_{\phi}$ to the eigenvalue, $\lambda$, in Eqs. (19), (20) and (22). Here $\tau_{\phi}$ is the dephasing time and it is assumed that $\tau_{\phi} \gg \tau_{e}$ in the weak-localization regime. The equivalent procedure in our formulation is to replace

$$
K^{(n)} \rightarrow K^{(n)} \exp \left(-\frac{(n+1) \tau_{e}}{\tau_{\phi}}\right) .
$$

Eqs. (18), (25) and (26) then yield

$$
g_{\mathrm{WL}}=-\frac{e^{2}}{\hbar} \frac{D \tau_{e}}{L^{2}} \int d \mathbf{r} \sum_{n} K^{(n)}(\mathbf{r}, \mathbf{r}) \exp \left(-\frac{n \tau_{e}}{\tau_{\phi}}\right) .
$$

In summary, Eqs. (23), (25) and (27) provide the tools to calculate the weak-localization conductance.

Eq. (27) has an appealing form. $g_{\mathrm{WL}}$ is expressed as a sum over closed random walks, consistent with the physical idea that it is due to the interference of closed paths and their time-reversed counterparts. The integral over $K^{(n)}$ gives the weight of $n$-bounce paths; the exponential factor shows that the contribution of very long paths is cut off by dephasing. For a different formulation of weak localization in terms of random walks see Ref. [25].

Let us explicitly calculate $g_{\mathrm{WL}}$. Successive integration over intermediate points shows

$$
K^{(n)}\left(\mathbf{r}, \mathbf{r}^{\prime}\right)=\frac{1}{(n+1) l_{e}^{2}} \exp \left(-\frac{\left|\mathbf{r}-\mathbf{r}^{\prime}\right|^{2}}{(n+1) l_{e}^{2}}\right),
$$

a well-known result. $K^{(n)}$ has the same form as $K^{(0)}$ but with $l_{e} \rightarrow \sqrt{n+1} l_{e}$ in agreement with the general proposition that the displacement of a random walker grows as the square root of the number of steps. Now

$$
\begin{aligned}
g_{\mathrm{WL}} & =-\frac{e^{2}}{\hbar} \frac{W}{L} \sum_{n=1}^{\infty} \frac{1}{n} \exp \left(-\frac{n \tau_{e}}{\tau_{\phi}}\right) \\
& \approx-\frac{e^{2}}{\hbar} \frac{W}{L} \ln \frac{\tau_{\phi}}{\tau_{e}} \\
& =-\frac{e^{2}}{\hbar} \frac{W}{L} \ln \frac{l_{\phi}}{l_{e}},
\end{aligned}
$$

a celebrated result [1] 3. Here we have introduced $l_{\phi}=$ $\sqrt{D \tau_{\phi}}$, the dephasing length, and made use of the inequality $\tau_{\phi} \gg \tau_{e}$. The logarithmic divergence of $g_{\mathrm{WL}}$ as $l_{\phi} \rightarrow \infty$ can be traced to the slow $1 / n$ decay of the weight of long paths. In a field theory formulation it indicates that the theory flows to strong coupling on long length scales but on shorter scales it is "asymptotically free" [26]. Provided that the cutoff $l_{\phi}$ is shorter than the localization length, perturbation theory is accurate. 


\section{B. Uniform Perpendicular Field}

We consider magnetic fields that are weak compared to impurity scattering. The precise condition we assume is $B \ll B_{e}$, where $B_{e}=\phi_{\mathrm{sc}} / 2 \pi l_{e}^{2}$ and $\phi_{\mathrm{sc}}=h / 2 e$ is the superconducting flux quantum. In this limit the classical paths are not modified by the magnetic field. Its main effect is to change the phase of the paths (the AharonovBohm effect). This has no effect on the classical Drude conductance, but the weak-localization correction, which is an interference effect, is suppressed in a manner analyzed in Ref. [3].

The starting point for this analysis is to assume that the effect of turning on a perpendicular field on the Green function is to multiply it by a phase factor:

$$
G_{B_{\perp}}^{R}\left(\mathbf{r}, \mathbf{r}^{\prime} ; E_{f}\right) \approx G_{B=0}^{R}\left(\mathbf{r}, \mathbf{r}^{\prime} ; E_{f}\right) \exp \left(i \frac{e}{\hbar} \int_{\mathbf{r}^{\prime}}^{\mathbf{r}} d \mathbf{l} \cdot \mathbf{A}\right) .
$$

Here $G_{B=0}$ is the disorder averaged Green function at zero field, Eq. (16); $G_{B_{\perp}}$, the Green function with the perpendicular magnetic field turned on; and the integral in the phase factor is evaluated along a straight line from $\mathbf{r}^{\prime}$ to $\mathbf{r}$. The zeroth order Cooperon becomes

$$
C_{B_{\perp}}^{(0)}\left(\mathbf{r}, \mathbf{r}^{\prime}\right)=C_{B=0}^{(0)}\left(\mathbf{r}, \mathbf{r}^{\prime}\right) \exp \left(i \frac{2 e}{\hbar} \int_{\mathbf{r}^{\prime}}^{\mathbf{r}} d \mathbf{l} \cdot \mathbf{A}\right) .
$$

Once again we are interested in the eigenfunctions of $C_{B_{\perp}}^{(0)}$ which obey

$$
\int d \mathbf{r}^{\prime} C_{B=0}^{(0)}\left(\mathbf{r}, \mathbf{r}^{\prime}\right) \exp \left(i \frac{2 e}{\hbar} \int_{\mathbf{r}^{\prime}}^{\mathbf{r}} d \mathbf{l} \cdot \mathbf{A}\right) Q_{\lambda}\left(\mathbf{r}^{\prime}\right)=\lambda Q_{\lambda}(\mathbf{r})
$$

For the slowly varying eigenfunctions Eq. (32) may be transformed into a differential equation by expanding both the phase factor and $Q_{\lambda}\left(\mathbf{r}^{\prime}\right)$ about $\mathbf{r}^{\prime}=\mathbf{r}$ to second order. The result is

$$
\left[D \tau_{e}\left(\nabla-i \frac{2 e}{\hbar} \mathbf{A}(\mathbf{r})\right)^{2}+1\right] Q_{\lambda}(\mathbf{r})=\left(\lambda+\frac{\tau_{e}}{\tau_{\phi}}\right) Q_{\lambda}(\mathbf{r}) .
$$

In Eq. (33) we have explicitly inserted a damping term $\tau_{e} / \tau_{\phi}$. The customary solution [3] is to construct the Cooperon from the Landau-level solutions to Eq. (33).

As in the previous subsection we now describe an alternative formulation. Consider the kernel

$$
K_{B_{\perp}}^{(0)}\left(\mathbf{r}, \mathbf{r}^{\prime}\right)=K_{B=0}^{(0)}\left(\mathbf{r}, \mathbf{r}^{\prime}\right) \exp \left(i \frac{2 e}{\hbar} \int_{\mathbf{r}^{\prime}}^{\mathbf{r}} d \mathbf{l} \mathbf{. A}\right)
$$

with $K_{B=0}^{(0)}$ given by Eq. (23). The slowly varying eigenfunctions of $K_{B_{\perp}}^{(0)}$ also obey Eq. (33); hence if we define $K_{B_{\perp}}$ via
$K_{B_{\perp}}\left(\mathbf{r}, \mathbf{r}^{\prime}\right)=K_{B_{\perp}}^{(0)}\left(\mathbf{r}, \mathbf{r}^{\prime}\right)+\int d \mathbf{r}^{\prime \prime} K_{B_{\perp}}^{(0)}\left(\mathbf{r}, \mathbf{r}^{\prime \prime}\right) K_{B_{\perp}}\left(\mathbf{r}^{\prime \prime}, \mathbf{r}^{\prime}\right)$,

then $K_{B_{\perp}}$ has the same long-distance behavior as $C_{B_{\perp}}$ and may be used in its place to calculate the conductance.

A useful expression for $K_{B_{\perp}}$ is obtained from Eq. (35) by iteration

$$
\begin{aligned}
K_{B_{\perp}}\left(\mathbf{r}, \mathbf{r}^{\prime}\right)= & \sum_{n=0}^{\infty} K_{B_{\perp}}^{(n)}\left(\mathbf{r}, \mathbf{r}^{\prime}\right) ; \\
K_{B_{\perp}}^{(n)}\left(\mathbf{r}, \mathbf{r}^{\prime}\right)= & \int d \mathbf{r}_{1} \ldots d \mathbf{r}_{n} K_{B=0}^{(0)}\left(\mathbf{r}, \mathbf{r}_{1}\right) \ldots \\
& \ldots K_{B=0}^{(0)}\left(\mathbf{r}_{n}, \mathbf{r}^{\prime}\right) \exp \left(i \frac{2 e}{\hbar} \int_{\mathbf{r}^{\prime}}^{\mathbf{r}} d \mathbf{l} \cdot \mathbf{A}\right) .
\end{aligned}
$$

The integral in the phase factor is evaluated along the path from $\mathbf{r}^{\prime}$ to $\mathbf{r}$ obtained by joining the points $\mathbf{r}^{\prime}, \mathbf{r}_{n}, \ldots, \mathbf{r}_{1}, \mathbf{r}$ with line segments in the given order. For $\mathbf{r}=\mathbf{r}^{\prime}$

$$
\begin{aligned}
K_{B_{\perp}}^{(n)}(\mathbf{r}, \mathbf{r})= & \int d \mathbf{r}_{1} \ldots d \mathbf{r}_{n} K_{B=0}^{(0)}\left(\mathbf{r}, \mathbf{r}_{1}\right) \ldots \\
& \ldots K_{B=0}^{(0)}\left(\mathbf{r}_{n}, \mathbf{r}\right) \exp \left(i 2 \pi \frac{\phi_{n}}{\phi_{\mathrm{sc}}}\right) .
\end{aligned}
$$

Here $\phi_{n}=$ flux through the polygon defined by the closed path from $\mathbf{r}$ to $\mathbf{r}$ and $\phi_{\mathrm{sc}}=h / 2 e$ is the superconducting flux quantum.

Using Eq. (18) and (36)

$$
g_{\mathrm{WL}}=-\frac{e^{2}}{\hbar} \frac{D \tau_{e}}{L^{2}} \int d \mathbf{r} \sum_{n=0}^{\infty} K_{B_{\perp}}^{(n)}(\mathbf{r}, \mathbf{r}) \exp \left(-(n+1) \frac{\tau_{e}}{\tau_{\phi}}\right) \text {. }
$$

In summary Eqs. (23), (37) and (38) provide the tools needed to calculate the perpendicular field magnetoconductance.

Let us now explicitly calculate the magnetoconductance. It is necessary to first evaluate $K_{B_{\perp}}^{(n)}(\mathbf{r}, \mathbf{r})$. The integrals are performed in Appendix B and will be used in later sections. For the present, more insight is gained by noticing that $K_{B_{\perp}}^{(n)}(\mathbf{r}, \mathbf{r})$ has a natural interpretation in terms of random walks.

Imagine that $n$-sided polygons are drawn on a plane starting from a fixed point $\mathbf{r}$. Let the probability density of drawing a polygon with vertices at $\mathbf{r}, \mathbf{r}_{1}, \mathbf{r}_{2}, \ldots, \mathbf{r}_{n}, \mathbf{r}$ be given by the Gaussian expression $\left[2 \pi l_{e}^{2}(n+\right.$ 1)] $K_{B=0}^{(0)}\left(\mathbf{r}, \mathbf{r}_{1}\right) K_{B=0}^{(0)}\left(\mathbf{r}_{1}, \mathbf{r}_{2}\right) \ldots K_{B=0}^{(0)}\left(\mathbf{r}_{n}, \mathbf{r}\right)$. The factor $2 \pi l_{e}^{2}(n+1)$ is included to normalize the distribution. Let $a$ denote the directed area of the polygon (defined in Appendix C). Then Eq. (37) may be rewritten as

$K_{B_{\perp}}^{n}(\mathbf{r}, \mathbf{r})=\frac{1}{2 \pi l_{e}^{2}(n+1)} \int_{-\infty}^{+\infty} d a P_{n+1}(a) \exp \left(2 \pi i \frac{B_{\perp} a}{\phi_{\mathrm{sc}}}\right)$. 
Here $P_{n}(a)$ is the probability distribution of the directed area of an $n$-sided polygon drawn from the Gaussian polygon ensemble defined above. Eq. (39) shows that $K_{B}^{(n)}(\mathbf{r}, \mathbf{r})$ is the Fourier transform of the distribution of directed areas. The directed area distribution is discussed in Appendix C. For $n \gg 1$

$$
P_{n}(a)=\frac{\pi}{2 n l_{e}^{2}} \operatorname{sech}^{2}\left(\frac{\pi a}{n l_{e}^{2}}\right)
$$

and its Fourier transform is

$$
K_{B_{\perp}}^{(n)}(\mathbf{r}, \mathbf{r})=\frac{B_{\perp}}{2 \phi_{\mathrm{sc}}}\left[\sinh \left(\frac{n \pi B_{\perp} l_{e}^{2}}{\phi_{\mathrm{sc}}}\right)\right]^{-1} .
$$

Eq. (41) is derived in Appendix B and is shown to be sufficiently accurate for our purposes for all $n$ provided $B_{\perp} \ll B_{e}$. For further discussion of the directed area distribution see Appendix C.

Substitution of Eq. (41) in Eq. (38) leads to

$$
g_{\mathrm{WL}}=-\frac{e^{2}}{\hbar} \frac{W}{L} \sum_{n=1}^{\infty} \frac{B_{\perp}}{2 B_{e}}\left[\sinh \left(\frac{n B_{\perp}}{2 B_{e}}\right)\right]^{-1} \exp \left(-\frac{n \tau_{e}}{\tau_{\phi}}\right) .
$$

Since $B_{\perp} \ll B_{e}$, the sum may be evaluated asymptotically.2 Using an integral representation of the digamma function [27]

$$
\psi(x)=\int_{0}^{\infty} d q\left(\frac{e^{-q}}{q}-\frac{e^{-x q}}{1-e^{-q}}\right),
$$

we obtain

$$
g_{\mathrm{WL}}=-\frac{1}{2 \pi^{2}} \frac{e^{2}}{\hbar} \frac{W}{L}\left[\ln \left(\frac{B_{e}}{B_{\perp}}\right)-\psi\left(\frac{1}{2}+\frac{B_{\phi}}{B_{\perp}}\right)\right] .
$$

Here $B_{\phi}$ is the field corresponding to a flux quantum through the phase-breaking length, $B_{\phi} \equiv \phi_{\mathrm{sc}} / 4 \pi l_{\phi}^{2}$. This is the ideal weak-localization lineshape derived by Hikami, Larkin and Nagaoka [3] and shown as the dotted curves in Figs. 1 and 2.

It is instructive to summarize the main points of the derivation. First the conductance is expressed as a sum of

\footnotetext{
${ }^{2}$ The lower limit of the sum requires delicate handling. Introduce a cutoff $N \gg 1$ but with $N B_{\perp} / B_{e} \ll 1$ and $N \tau_{e} / \tau_{\phi} \ll 1$. The sum over $n$ from 1 to $N$ is approximately $\ln N$ since the summand is approximately $1 / n$ over this range. The sum from $N$ to infinity may be turned into an integral in the limit $B_{\perp} / 2 B_{e} \rightarrow 0$. The integral diverges logarithmically at its lower limit. The divergence may be extracted by integration by parts. This cancels the log dependence of the first sum on the arbitrary cutoff $N$ and leaves a finite expression that can be expressed in terms of the digamma function using Eq. (43).
}

closed random walks, Eq. (38). Eq. (37) shows that the contribution of a fixed random walk oscillates with the enclosed flux with a period $\phi_{\mathrm{sc}}$, revealing the AharonovBohm origin of the weak-localization magnetoresistance. The contribution of all $n$-bounce paths is the Fourier transform of their directed area distribution; it decays exponentially for $B_{\perp} \gg \phi_{\mathrm{sc}} / n l_{e}^{2}$ [see Eq. (42)]. Summing over $n$ leads to the gentler decay described by Eq. (44).

\section{SHORT RANGE ROUGHNESS}

In this section we assume that the distance over which the roughness is correlated is short compared to the elastic mean free path $\left(L \ll l_{e}\right)$. Under this circumstance the effect of turning on an in-plane magnetic field is comparatively straightforward: it enhances the dephasing rate and hence produces "homogeneous broadening" of the weak-localization lineshape.

For simplicity we first assume that the applied field lies in the plane of the interface. According to the analysis of Section II, the interface electrons see a uniform inplane field as a random perpendicular field. The typical strength of the random field is $B_{g} \sim B_{\|} \Delta / L$ [see Eqs. (5) and (12)]. We assume that the random magnetic field is weak compared to impurity scattering $\left(B_{g} \ll B_{e}\right)$, a condition that is well satisfied in practice. As in the previous section, the only effect of the magnetic field in this limit is to multiply the Green function by a phase factor, so the zeroth-order Cooperon becomes

$$
C_{B_{\|}}^{(0)}\left(\mathbf{r}, \mathbf{r}^{\prime}\right)=C_{B=0}^{(0)}\left(\mathbf{r}, \mathbf{r}^{\prime}\right) \exp \left(i \frac{2 e}{\hbar} \int_{\mathbf{r}^{\prime}}^{\mathbf{r}} d \mathbf{l} . \mathbf{A}_{g}\right) .
$$

The Cooperon is determined in principle by Eqs. (17) and (45); our objective is to average it over the interface roughness.

To this end it is convenient to formally solve Eq. (17) by iteration:

$$
\begin{aligned}
C_{B_{\|}}(\mathbf{r}, \mathbf{r})= & \sum_{n=0}^{\infty} C_{B_{\|}}^{(n)}(\mathbf{r}, \mathbf{r}) ; \\
C_{B_{\|}}^{(n)}(\mathbf{r}, \mathbf{r})= & \int d \mathbf{r}_{1} \ldots d \mathbf{r}_{n} C_{B=0}^{(0)}\left(\mathbf{r}, \mathbf{r}_{1}\right) \ldots \\
& \ldots C_{B=0}^{(0)}\left(\mathbf{r}_{n}, \mathbf{r}\right) \exp \left(i 2 \pi \frac{\phi_{g}}{\phi_{\mathrm{sc}}}\right) ; \\
\phi_{g}= & \int_{\mathbf{r}}^{\mathbf{r}} d \mathbf{l} \cdot \mathbf{A}_{g} .
\end{aligned}
$$

The integral in Berry's phase factor, $\phi_{g}$, is to be evaluated around the perimeter of the polygon obtained by joining $\mathbf{r}, \mathbf{r}_{1}, \ldots, \mathbf{r}_{n}, \mathbf{r}$ in that order. We average the Berry phase factor using the gauge given by Eq. (8) and assuming that $\mathcal{Z}_{g}(x, y)$ is a Gaussian random surface with statistics given by Eq. (12). To avoid digression we shall return 
to the evaluation of this average below. For the moment we give the result for $L \ll l_{e}$ :

$$
\begin{aligned}
& \left\langle\exp \left(i 2 \pi \frac{\phi_{g}}{\phi_{\mathrm{sc}}}\right)\right\rangle_{\text {rough }} \approx \\
& \exp \left(-2 \sqrt{\pi} \frac{e^{2}}{\hbar^{2}} B_{\|}^{2} \Delta^{2} L\left[\frac{\left(y-y_{1}\right)^{2}}{\left|\mathbf{r}-\mathbf{r}_{1}\right|}+\ldots+\frac{\left(y_{n}-y\right)^{2}}{\left|\mathbf{r}_{n}-\mathbf{r}\right|}\right]\right) .
\end{aligned}
$$

From Eqs. (46) and (47) we obtain the average Cooperon,

$$
\begin{aligned}
\mathcal{C}_{B_{\|}}(\mathbf{r}, \mathbf{r}) & =\sum_{n=0}^{\infty} \mathcal{C}_{B_{\|}}^{(n)}(\mathbf{r}, \mathbf{r}) \\
\mathcal{C}_{B_{\|}}^{(n)}(\mathbf{r}, \mathbf{r}) & =\int d \mathbf{r}_{1} \ldots d \mathbf{r}_{n} \mathcal{C}_{B_{\|}}^{(0)}\left(\mathbf{r}, \mathbf{r}_{1}\right) \ldots \mathcal{C}_{B_{\|}}^{(0)}\left(\mathbf{r}_{n}, \mathbf{r}\right) \\
\mathcal{C}_{B_{\|}}^{(0)}\left(\mathbf{r}, \mathbf{r}^{\prime}\right) & =C_{B=0}^{(0)}\left(\mathbf{r}, \mathbf{r}^{\prime}\right) \exp \left(-2 \sqrt{\pi} \frac{e^{2}}{\hbar^{2}} B_{\|}^{2} \Delta^{2} L \frac{\left(y-y^{\prime}\right)^{2}}{\left|\mathbf{r}-\mathbf{r}^{\prime}\right|}\right)
\end{aligned}
$$

For brevity $\left\langle C_{B_{\|}}(\mathbf{r}, \mathbf{r})\right\rangle_{\text {rough }}$ is written as $\mathcal{C}_{B_{\|}}(\mathbf{r}, \mathbf{r})$.

Evidently Eq. (48) is the formal iterative solution to the integral equation

$$
\mathcal{C}_{B_{\|}}\left(\mathbf{r}, \mathbf{r}^{\prime}\right)=\mathcal{C}_{B_{\|}}^{(0)}\left(\mathbf{r}, \mathbf{r}^{\prime}\right)+\int d \mathbf{r}^{\prime \prime} \mathcal{C}_{B_{\|}}^{(0)}\left(\mathbf{r}, \mathbf{r}^{\prime \prime}\right) \mathcal{C}_{B_{\|}}\left(\mathbf{r}^{\prime \prime}, \mathbf{r}^{\prime}\right)
$$

In principle the roughness averaged Cooperon is determined by Eq. (49) with $\mathcal{C}_{B_{\|}}^{(0)}\left(\mathbf{r}, \mathbf{r}^{\prime}\right)$ given by Eqs. (21) and (48). The expression for $\mathcal{C}_{B_{\|}}^{(0)}\left(\mathbf{r}, \mathbf{r}^{\prime}\right)$ shows that after averaging over roughness, the effect of the in-plane field is to multiply the zeroth-order Cooperon by an exponential factor.

To solve Eq. (49) it is most convenient to look for eigenfunctions of $C_{B_{\|}}^{(0)}$ which obey

$$
\begin{aligned}
& \int d \mathbf{r}^{\prime} C_{B=0}^{(0)}\left(\mathbf{r}, \mathbf{r}^{\prime}\right) \exp \left(-2 \sqrt{\pi} \frac{e^{2}}{\hbar^{2}} B_{\|}^{2} \Delta^{2} L \frac{\left(y-y^{\prime}\right)^{2}}{\left|\mathbf{r}-\mathbf{r}^{\prime}\right|}\right) Q_{\lambda}\left(\mathbf{r}^{\prime}\right) \\
& =\lambda Q_{\lambda}(\mathbf{r}) .
\end{aligned}
$$

$\mathcal{C}_{B_{\|}}$may be expanded in terms of these eigenfunctions as in Eq. (20). We are interested in eigenfunctions that vary slowly compared to $l_{e}$. For these eigenfunctions Eq. (50) may be transformed into a diffusion equation by expanding $Q_{\lambda}$ and the exponential factor in powers of $\mathbf{r}-\mathbf{r}^{\prime}$. Keeping the leading terms (second order in $Q_{\lambda}$ and first-order in the exponential factor) we obtain

$$
\left(1+D \tau_{e} \nabla^{2}\right) Q_{\lambda}(\mathbf{r})=\left(\lambda+\frac{\tau_{e}}{\tau_{\phi}}+\frac{\tau_{e}}{\tau_{\|}}\right) Q_{\lambda}(\mathbf{r}) .
$$

In Eq. (51) we have put a dephasing term $\tau_{e} / \tau_{\phi}$ by hand. The in-plane field is responsible for the $\tau_{e} / \tau_{\|}$term. Here $\tau_{\|}$is defined by

$$
\begin{aligned}
\frac{\tau_{e}}{\tau_{\|}} & =2 \sqrt{\pi} \frac{e^{2}}{\hbar^{2}} B_{\|}^{2} \Delta^{2} L \int d \mathbf{r}^{\prime} C_{B=0}^{(0)}\left(\mathbf{r}, \mathbf{r}^{\prime}\right) \frac{\left(y-y^{\prime}\right)^{2}}{\left|\mathbf{r}-\mathbf{r}^{\prime}\right|} \\
& \approx \sqrt{\pi} \frac{e^{2}}{\hbar^{2}} B_{\|}^{2} \Delta^{2} L l_{e}
\end{aligned}
$$

Eq. (51) shows that, upon averaging, the only effect of an in-plane magnetic field on the long-distance behavior of the Cooperon, and hence on the conductance, is to enhance the dephasing rate by

$$
\frac{1}{\tau_{\phi}} \rightarrow \frac{1}{\tau_{\phi}}+\frac{1}{\tau_{\|}}
$$

where $\tau_{\|}$is given by Eq. (52).

The form of $\tau_{\|}^{-1}$ in Eq. (52) can be obtained from the following simple argument. We wish to find the typical phase associated with an n-sided random polygon due to the random vector potential. Consider a single side of the polygon: it has typical length $l_{e}$, and the vector potential is approximately constant over segments of length $L$ but of either $\operatorname{sign}-A \sim \pm B_{\|} \Delta$. Then the typical phase accumulated on this side is $\operatorname{rms}\left[(2 e / \hbar) \int \mathbf{A} \cdot d \mathbf{l}\right] \sim$ $\left.(2 e / \hbar) \sqrt{l_{e} / L}\right) B_{\|} \Delta L$. Since the phase along each side is uncorrelated, the typical total phase is $\operatorname{rms}\left[\phi_{\mathrm{n}-\text { gon }}\right] \sim$ $\left.\sqrt{n}(2 e / \hbar) \sqrt{l_{e} / L}\right) B_{\|} \Delta L$. The contribution of $\mathrm{n}$-gons will be cutoff when this phase is of order 1 , giving the condition for $\tau_{\|}^{-1}$. This gives the condition for $\tau_{\|}^{-1}$ if we set $n \rightarrow \tau_{\|} / \tau_{e}$. We find $\tau_{e} / \tau_{\|} \sim 4(e / \hbar)^{2} B_{\|}^{2} \Delta^{2} L l_{e}$, the same form as Eq. (52).

Now we indicate how to generalize this analysis when the applied magnetic field has a non-zero perpendicular component. Assuming this component is weak compared to impurity scattering $\left(B_{\perp} \ll B_{e}\right)$, an Aharonov-Bohm phase factor must be included in Eqs. (45) and (46). After averaging over interface roughness $\mathcal{C}_{B_{\|}, B_{\perp}}$ obeys Eq. (49) with

$$
\begin{aligned}
\mathcal{C}_{B_{\|}, B_{\perp}}^{(0)}\left(\mathbf{r}, \mathbf{r}^{\prime}\right)= & C_{B=0}^{(0)}\left(\mathbf{r}, \mathbf{r}^{\prime}\right) \exp \left(i \frac{2 e}{\hbar} \int_{\mathbf{r}^{\prime}}^{\mathbf{r}} d \mathbf{l} . \mathbf{A}\right) \\
& \times \exp \left(-2 \sqrt{\pi} \frac{e^{2}}{\hbar^{2}} B_{\|}^{2} \Delta^{2} L \frac{\left(y-y^{\prime}\right)^{2}}{\left|\mathbf{r}-\mathbf{r}^{\prime}\right|}\right) .
\end{aligned}
$$

Keeping track of the phase factor, we find that the slowly varying eigenfunctions of $\mathcal{C}_{B_{\|}, B_{\perp}}^{(0)}$ obey Eq. (33) with the substitution given in Eq. (53).

Hence we arrive at the central result of this section. For short-range correlated roughness, application of an in-plane field increases the dephasing rate according to Eq. (53) but does not otherwise alter the weaklocalization lineshape. The dependence of the conductance on the perpendicular field is still given by Eq. (44) but with

$$
B_{\phi} \rightarrow B_{\phi}+\pi^{3 / 2} \frac{e B_{\|}^{2}}{h} \frac{\Delta^{2} L}{l_{e}}
$$


Since the form of the lineshape is not changed, this effect is analogous to homogeneous broadening in magnetic resonance and atomic physics.

The idea that an in-plane field produces homogeneous broadening of the line was anticipated by the authors of Refs. [5.6. Our result differs in two important respects: First, we find that homogeneous broadening occurs only if the roughness is correlated over short distances $(L \ll$ $l_{e}$ ); and second, the dependence of $\tau_{\|}$on the interface roughness parameters that we derive [Eq. (52)] is quite different from that conjectured in Ref. [6].

Fig. 6 shows that Eqs. (44) and (55) provide a good fit to data with essentially no adjustable parameters. More details are given in Section VI.

To complete the analysis we must now average Berry's phase factor $\exp \left(i 2 \pi \phi_{g} / \phi_{\text {sc }}\right)$ over interface roughness to obtain Eq. (47). Recall that the distribution of a linear combination of correlated Gaussian random variables is also Gaussian. The distribution of $\phi_{g}$ is therefore Gaussian. Evidently it has zero mean since we have assumed $\left\langle\mathcal{Z}_{g}(x, y)\right\rangle_{\text {rough }}=0$. For a Gaussian random variable, $s$, with zero mean it is easy to show

$$
\langle\exp (i \xi s)\rangle=\exp \left(-\frac{1}{2} \xi^{2}\left\langle s^{2}\right\rangle\right) .
$$

Hence averaging Berry's phase factor reduces to the calculation of the variance of the phase. Explicitly we must calculate

$$
\left\langle\phi_{g}^{2}\right\rangle_{\mathrm{rough}}=\sum_{i=1}^{n+1} \sum_{j=1}^{n+1}\left\langle\int d \mathbf{l}_{i} \cdot \mathbf{A}_{g} \int d \mathbf{l}_{j} \cdot \mathbf{A}_{g}\right\rangle_{\text {rough }} .
$$

Here $\int d \mathbf{l}_{i} \cdot \mathbf{A}_{g}$ denotes the line integral along the edge joining $\mathbf{r}_{i-1}$ to $\mathbf{r}_{i}$ (with the understanding that $\mathbf{r}_{0}=$ $\left.\mathbf{r}_{n+1}=\mathbf{r}\right)$.

Let us first evaluate the diagonal terms in Eq. (57). Let the $i^{\text {th }}$ edge make an angle $\theta$ with the $x$-axis and let $s$ be the length parameter along that edge. Using Eq. (8) for $\mathbf{A}_{g}$ and Eq. (12) for the correlation of $\mathcal{Z}_{g}(x, y)$ we obtain

$$
\begin{aligned}
& \left\langle\left(\int d \mathbf{l}_{i} \cdot \mathbf{A}_{g}\right)^{2}\right\rangle_{\text {rough }}= \\
& B_{\|}^{2} \Delta^{2} \sin ^{2} \theta \int_{0}^{\left|\mathbf{r}_{i}-\mathbf{r}_{i-1}\right|} d s \int_{0}^{\left|\mathbf{r}_{i}-\mathbf{r}_{i-1}\right|} d s^{\prime} \exp \left[-\left(\frac{s-s^{\prime}}{L}\right)^{2}\right] \\
& \approx \sqrt{\pi} B_{\|}^{2} \Delta^{2} L \frac{\left(y_{i}-y_{i-1}\right)^{2}}{\left|\mathbf{r}_{i}-\mathbf{r}_{i-1}\right|} .
\end{aligned}
$$

We have assumed that the edge is much longer than the correlation length $L$. Since the length of a typical edge is $l_{e}$, this is justified in the short range limit $L \ll l_{e}$. We have also used $\left|\mathbf{r}_{i}-\mathbf{r}_{i-1}\right| \sin \theta=y_{i}-y_{i-1}$.

Evidently the only important off diagonal terms in Eq. (57) are those for which the two edges pass within a correlation length of each other. The contribution of such edge pairs to the sum will be smaller than the diagonal terms, typically by a factor of $L / l_{e}$ (except in the rare circumstance that the two edges meet at a glancing angle and overlap for a considerable part of their length). Furthermore, the number of contributing off-diagonal terms is comparable to $n$, the number of diagonal terms; hence the contribution of the off-diagonal terms may be safely neglected in the short range limit $L \ll l_{e}$.

That the number of important off-diagonal terms is comparable to $n$ can be established by an argument commonly used in polymer physics [28]. Suppose that line segments of typical length $l_{e}$ are drawn at random on a plane with a density $\rho$ segments per unit area. If a fresh segment of length $l_{e}$ is now drawn on this plane, typically it will suffer $\sim \rho l_{e}^{2}$ intersections. Working in units where $l_{e}=1$, the area of a typical polygon in Eq. (46) is $n$ and hence the density of edges $\rho \sim n / n=1$. Each edge therefore typically encounters one intersection and the total number of intersections is comparable to $n$.

Adding the diagonal contributions [Eq. (58)] and neglecting the off-diagonal ones we obtain

$$
\left\langle\phi_{g}^{2}\right\rangle_{\text {rough }} \approx \sqrt{\pi} B_{\|}^{2} \Delta^{2} L\left[\frac{\left(y_{1}-y\right)^{2}}{\left|\mathbf{r}_{1}-\mathbf{r}\right|}+\ldots+\frac{\left(y-y_{n}\right)^{2}}{\left|\mathbf{r}-\mathbf{r}_{n}\right|}\right] .
$$

Use of Eqs. (56) and (59) finally leads to Eq. (47).

\section{LONG RANGE ROUGHNESS}

We now consider the roughness correlation length to be long compared to the mean free path $\left(L \gg l_{e}\right)$. This circumstance is more difficult to analyze; hence, the discussion is limited to the circumstance that the in-plane magnetic field is weak in a sense made precise below. Roughly it is assumed that the random Berry phase is small for all contributing paths. The experiments of Refs. [5] meet this condition for most part. For simplicity, we first assume the applied field lies in the plane of the interface, $B_{\perp}=0$ (Section Va). In the next subsection no restriction is placed on $B_{\perp}$ (other than $B_{\perp} \ll B_{e}$ ).

\section{A. Zero Perpendicular Field Applied}

Since $B_{g} \ll B_{e}$ is automatically ensured by the weak field condition on $B_{\|}$that we will impose below, the Cooperon is given by Eq. (45). Since the Berry phase factor varies on the scale of $L \gg l_{e}$, we may work with $K$ instead of $C$ and write

\footnotetext{
${ }^{3}$ This is more stringent than the condition $B_{g} \sim B_{\|} \Delta / L \ll$ $B_{e}$ imposed in the previous section [see discussion following Eq. (45)].
} 


$$
K_{B_{\|}}^{(0)}\left(\mathbf{r}, \mathbf{r}^{\prime}\right)=K_{B=0}^{(0)}\left(\mathbf{r}, \mathbf{r}^{\prime}\right) \exp \left(i \frac{2 e}{\hbar} \int_{\mathbf{r}^{\prime}}^{\mathbf{r}} d \mathbf{l} . \mathbf{A}_{g}\right)
$$

in place of Eq. (45) and

$$
\begin{aligned}
K_{B_{\|}}(\mathbf{r}, \mathbf{r})= & \sum_{n=0}^{\infty} K_{B_{\|}}^{(n)}(\mathbf{r}, \mathbf{r}) ; \\
K_{B_{\|}}^{(n)}(\mathbf{r}, \mathbf{r})= & \int d \mathbf{r}_{1} \ldots d \mathbf{r}_{n} K_{B=0}^{(0)}\left(\mathbf{r}, \mathbf{r}_{1}\right) \ldots \\
& \ldots K_{B=0}^{(0)}\left(\mathbf{r}_{n}, \mathbf{r}\right) \exp \left(i 2 \pi \frac{\phi_{g}}{\phi_{\mathrm{sc}}}\right) ; \\
\phi_{g}= & \int_{\mathbf{r}}^{\mathbf{r}} d \mathbf{l} \cdot \mathbf{A}_{g}
\end{aligned}
$$

in place of Eq. (46). Note that we could not make this replacement in the previous section since the Berry phase fluctuated rapidly on the scale of $l_{e}$. Our objective now is to average $K_{B_{\|}}$over interface roughness and then use Eq. (27) to evaluate the conductance.

The Berry phase factor is a Gaussian random variable so it is easy to perform the average. Using Eq. (8) for the vector potential, Eq. (12) for its statistics and Eq. (56), we obtain

$$
\begin{aligned}
\mathcal{K}_{B_{\|}}^{(n)}(\mathbf{r}, \mathbf{r})= & \int d \mathbf{r}_{1} \ldots d \mathbf{r}_{n} K_{B=0}^{(0)}\left(\mathbf{r}, \mathbf{r}_{1}\right) \ldots \\
& \ldots K_{B=0}^{(0)}\left(\mathbf{r}_{n}, \mathbf{r}\right) \exp \left[-V\left(\mathbf{r}, \mathbf{r}_{1}, \ldots, \mathbf{r}_{n}\right)\right]
\end{aligned}
$$

with

$$
\begin{aligned}
& V\left(\mathbf{r}, \mathbf{r}_{1}, \ldots, \mathbf{r}_{n}\right)= \\
& \frac{4 e^{2} B_{\|}^{2} \Delta^{2}}{\hbar^{2}} \sum_{j=1}^{n} \sum_{k=1}^{n}\left(y_{j}-y_{j-1}\right)\left(y_{k}-y_{k-1}\right) \\
& \quad \times \exp \left[-\frac{1}{4 L^{2}}\left(\mathbf{r}_{j}+\mathbf{r}_{j-1}-\mathbf{r}_{k}-\mathbf{r}_{k-1}\right)^{2}\right] .
\end{aligned}
$$

Here $\mathcal{K}^{(n)}=\left\langle K^{(n)}\right\rangle_{\text {rough }}$ and $\mathbf{r}_{0}=\mathbf{r}_{n+1}=\mathbf{r}$. $\mathcal{K}^{(n)}$ is thus an integral over $(n+1)$-bounce closed random walks just as it is at zero field but the weight of each polygon is no longer Gaussian. The links of the polygon now "interact", and the interaction, given by Eq. (63), is anisotropic and long-ranged.

$\mathcal{K}^{(n)}$ is difficult to calculate. For weak in-plane magnetic fields it is sufficient to analyze the interaction perturbatively. To first order

$$
\begin{aligned}
\delta \mathcal{K}_{B_{\|}}^{(n)}(\mathbf{r}, \mathbf{r}) \equiv & \mathcal{K}_{B_{\|}}^{(n)}(\mathbf{r}, \mathbf{r})-K_{B=0}^{(n)}(\mathbf{r}, \mathbf{r}) \\
= & -\int d \mathbf{r}_{1} \ldots d \mathbf{r}_{n} K_{B=0}^{(0)}\left(\mathbf{r}, \mathbf{r}_{1}\right) \ldots \\
& \ldots K_{B=0}^{(0)}\left(\mathbf{r}_{n}, \mathbf{r}\right) V\left(\mathbf{r}, \mathbf{r}_{1}, \ldots, \mathbf{r}_{n}\right)
\end{aligned}
$$

$V\left(\mathbf{r}, \mathbf{r}_{1}, \ldots, \mathbf{r}_{n}\right)$ is a sum over pairs of links according to Eq. (63). To illustrate the evaluation of Eq. (64) focus on the particular term for which $j=1$ and $k=3,4, \ldots, n$.
It is convenient to perform the integrals over the end points of the $j^{\text {th }}$ and $k^{\text {th }}$ links last. Integrating over the remaining intermediate points yields

$$
\begin{aligned}
\delta \mathcal{K}_{B_{\|}}^{(n)}(\mathbf{r}, \mathbf{r})= & \frac{1}{\pi^{2}} \int d \mathbf{r} d \mathbf{r}_{1} d \mathbf{r}_{k-1} d \mathbf{r}_{k} \\
& \times \frac{1}{(n+1-k)(k-2)}\left(y_{1}-y\right)\left(y_{k}-y_{k-1}\right) \\
& \times \exp \left[-\frac{\left(\mathbf{r}-\mathbf{r}_{k}\right)^{2}}{2 l_{e}^{2}(n+1-k)}-\frac{\left(\mathbf{r}_{k-1}-\mathbf{r}_{1}\right)^{2}}{2 l_{e}^{2}(k-2)}\right] \\
& \times \exp \left[-\frac{\left(\mathbf{r}_{k}-\mathbf{r}_{k-1}\right)^{2}}{2 l_{e}^{2}}-\frac{\left(\mathbf{r}_{1}-\mathbf{r}\right)^{2}}{2 l_{e}^{2}}\right] \\
& \times \exp \left[\frac{1}{4 L^{2}}\left(\mathbf{r}+\mathbf{r}_{1}-\mathbf{r}_{k-1}-\mathbf{r}_{k}\right)^{2}\right]
\end{aligned}
$$

This is a low-dimensional Gaussian integral and can be explicitly evaluated.

Proceeding in this manner we obtain

$$
\begin{aligned}
\delta \mathcal{K}_{B_{\|}}^{(n)}(\mathbf{r}, \mathbf{r}) & =\left(\frac{e B_{\|} \Delta}{\hbar}\right)^{2}\left[f_{1}(n, d)+f_{2}(n, d)+f_{3}(n, d)\right] \\
f_{1}(n, d) & \approx \frac{n(1+n d)}{16 d^{2}} \sum_{k=3}^{n} \frac{1}{\left(k^{2}-n k-[n / 4 d]\right)^{2}} \\
f_{2}(n, d) & \approx \frac{1+n d}{n} \\
f_{3}(n, d) & \approx 1
\end{aligned}
$$

with $d=l_{e}^{2} /\left(2 L^{2}\right)$. The three contributions to $\mathcal{K}^{(n)}$ arise from the interaction of disconnected links, adjacent links, and from the self-interaction of links, respectively. We have used $n \gg 1$ and $d \ll 1$ to simplify Eq. (66). This is justified because weak-localization is dominated by long paths and we are concerned with long-range correlated roughness in this section.

Using Eq. (27) for the conductance and Eq. (66) for $\delta \mathcal{K}^{(n)}$, after considerable simplification we obtain the parallel field magnetoconductance

$$
\delta g=-\frac{e^{2}}{\hbar} \frac{1}{\phi_{\mathrm{sc}}^{2}}\left(B_{\|} \Delta L\right)^{2} f(\alpha)
$$

with $\alpha=l_{\phi}^{2} / L^{2}$ and

$$
\begin{aligned}
f(\alpha)= & \alpha \int_{0}^{\infty} d x e^{-x} \\
& \times\left(1+\frac{1}{2[x \alpha(1+x \alpha)]^{1 / 2}} \ln \frac{\sqrt{1+x \alpha}-\sqrt{x \alpha}}{\sqrt{1+x \alpha}+\sqrt{x \alpha}}\right) .
\end{aligned}
$$

It is striking that $\delta g$ is independent of the mean free path $l_{e}$. In appropriate units it is a product of two factors: the flux through an area $\Delta L$, determined entirely by the geometry of the rough interface, and $f(\alpha) . f(\alpha)$ describes the crossover as $l_{\phi}$ is varied relative to $L$. It is plotted in Fig. 4 and has the asymptotic behavior 


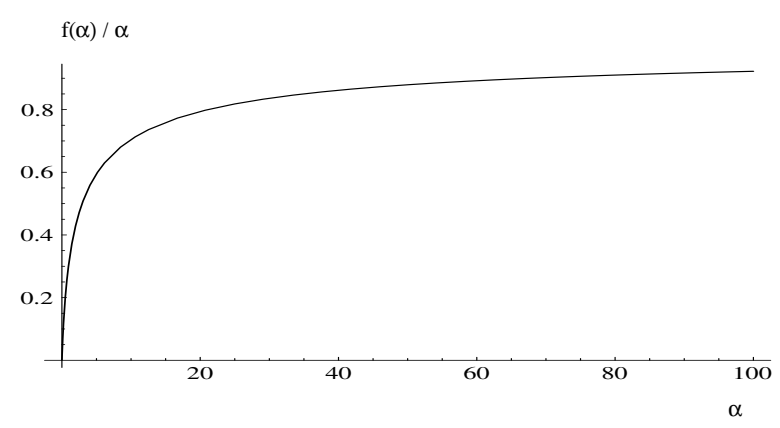

FIG. 4. Plot of $f(\alpha) / \alpha$ as a function of $\alpha=l_{\phi}^{2} / L^{2}$. $f(\alpha)$ describes the dependence of the in-plane magnetoresistance, at zero perpendicular field, on the roughness correlation length, $L$ (see Eq. 67).

$$
\begin{aligned}
f(\alpha) & \approx \alpha \text { for } \alpha \gg 1 \\
& \approx \frac{2}{3} \alpha^{2} \text { for } \alpha \ll 1 .
\end{aligned}
$$

Substituting Eq. (69) in Eq. (67) gives the asymptotic behavior of the parallel field magnetoconductance

$$
\begin{aligned}
\delta g & \approx-\frac{e^{2}}{\hbar} \frac{B_{\|}^{2} \Delta^{2} l_{\phi}^{2}}{\phi_{\mathrm{sc}}^{2}} \text { for } l_{\phi} \gg L \\
& \approx-\frac{2}{3} \frac{e^{2}}{\hbar} \frac{B_{\|}^{2} \Delta^{2} l_{\phi}^{4}}{\phi_{\mathrm{sc}}^{2} L^{2}} \text { for } L \gg l_{\phi}
\end{aligned}
$$

These formulae have a simple interpretation. The random magnetic field has a typical magnitude $B_{\|} \Delta / L$ and is correlated over a distance $L$. For $L \gg l_{\phi}$, the typical flux through an area $l_{\phi}^{2}$ is therefore $\left(B_{\|} \Delta / L\right) l_{\phi}^{2}$; for $L \ll l_{\phi}$, it is $B_{\|} \Delta l_{\phi}$ (see footnote ${ }^{4}$ ). Eq. (70) then shows that $\delta g$ (in units of $e^{2} / h$ ) is the square of the flux through a phase-coherent region in units of the flux quantum.

\section{B. Perpendicular Field Applied}

The calculation with a perpendicular field applied closely parallels the calculation at $B_{\perp}=0$. We write

$$
K_{B_{\perp}, B_{\|}}^{(0)}\left(\mathbf{r}, \mathbf{r}^{\prime}\right)=K_{B=0}^{(0)}\left(\mathbf{r}, \mathbf{r}^{\prime}\right) \exp \left[i \frac{2 e}{\hbar} \int_{\mathbf{r}^{\prime}}^{\mathbf{r}} d \mathbf{l} \cdot\left(\mathbf{A}_{g}+\mathbf{A}\right)\right]
$$

in place of Eq. (60) and

\footnotetext{
${ }^{4}$ The area $l_{\phi}^{2}$ can be broken up into $l_{\phi}^{2} / L^{2}$ correlated squares of area $L^{2}$. The typical flux through each square is $\left(B_{\|} \Delta / L\right) L^{2}$. Since it can be of either sign, the flux through the total area $l_{\phi}^{2}$ grows as the square root of the number of squares.
}

$$
\begin{aligned}
K_{B_{\perp}, B_{\|}}(\mathbf{r}, \mathbf{r})= & \sum_{n=0}^{\infty} K_{B_{\perp}, B_{\|}}^{(n)}(\mathbf{r}, \mathbf{r}) ; \\
K_{B_{\perp}, B_{\|}}^{(n)}(\mathbf{r}, \mathbf{r})= & \int d \mathbf{r}_{1} \ldots d \mathbf{r}_{n} K_{B=0}^{(0)}\left(\mathbf{r}, \mathbf{r}_{1}\right) \\
& \ldots K_{B=0}^{(0)}\left(\mathbf{r}_{n}, \mathbf{r}\right) \ldots \\
& \ldots \exp \left(i 2 \pi \frac{\phi_{g}}{\phi_{\mathrm{sc}}}\right) \exp \left(i 2 \pi \frac{\phi_{\perp}}{\phi_{\mathrm{sc}}}\right) ; \\
\phi_{g}= & \int_{\mathbf{r}}^{\mathbf{r}} d \mathbf{l} \cdot \mathbf{A}_{g} ; \\
\phi_{\perp}= & \int_{\mathbf{r}}^{\mathbf{r}} d \mathbf{l} \cdot \mathbf{A}
\end{aligned}
$$

in place of Eq. (61). Here $\phi_{\perp}$ is the Aharonov-Bohm flux through the polygon that goes from $\mathbf{r}$ to $\mathbf{r}$ via $\mathbf{r}_{1} \ldots \mathbf{r}_{n}$ due to the applied perpendicular field; $\phi_{g}$ is the Berry phase around the same polygon.

The Berry phase factor is a Gaussian random variable. Performing the average as in the previous subsection yields

$$
\begin{aligned}
\mathcal{K}_{B_{\perp}, B_{\|}}^{(n)}(\mathbf{r}, \mathbf{r})= & \int d \mathbf{r}_{1} \ldots d \mathbf{r}_{n} K_{B=0}^{(0)}\left(\mathbf{r}, \mathbf{r}_{1}\right) \ldots \\
& \ldots K_{B=0}^{(0)}\left(\mathbf{r}_{n}, \mathbf{r}\right) \exp \left[-V\left(\mathbf{r}, \mathbf{r}_{1}, \ldots, \mathbf{r}_{n}\right)\right] \\
& \times \exp \left(i 2 \pi \frac{\phi_{\perp}}{\phi_{\mathrm{sc}}}\right)
\end{aligned}
$$

Here $\mathcal{K}^{(n)}=\left\langle K^{(n)}\right\rangle_{\text {rough }}$ and $V$ is given by Eq. (63). As in Section IIIB, $\mathcal{K}^{(n)}$ is the Fourier transform of the directed area distribution of $n$-sided polygons; but the weight of the polygons is no longer Gaussian. The links of the polygons now "interact" and the interaction given by Eq. (63) is anisotropic and long-ranged.

For weak in-plane fields a full analysis of $\mathcal{K}^{(n)}$ is not needed. It is sufficient to analyze the interaction perturbatively. To first order

$$
\begin{aligned}
\delta \mathcal{K}_{B_{\perp}, B_{\|}}^{(n)}(\mathbf{r}, \mathbf{r}) \equiv & \mathcal{K}_{B_{\perp}, B_{\|}}^{(n)}(\mathbf{r}, \mathbf{r})-K_{B_{\perp}}^{(n)}(\mathbf{r}, \mathbf{r}) \\
= & -\int d \mathbf{r}_{1} \ldots d \mathbf{r}_{n} K_{B=0}^{(0)}\left(\mathbf{r}, \mathbf{r}_{1}\right) \\
& \ldots K_{B=0}^{(0)}\left(\mathbf{r}_{n}, \mathbf{r}\right) V\left(\mathbf{r}, \ldots, \mathbf{r}_{n}\right) \\
& \times \exp \left(i \frac{2 \pi \phi_{\perp}}{\phi_{\mathrm{sc}}}\right)
\end{aligned}
$$

$V\left(\mathbf{r}, \mathbf{r}_{1}, \ldots, \mathbf{r}_{n}\right)$ is a sum over pairs of links according to Eq. (63). To evaluate Eq. (74) it is convenient to focus on the particular term corresponding to the $j^{\text {th }}$ and $k^{\text {th }}$ links. As in the corresponding evaluation of Eq. (64), it is convenient to perform the integrals over the end points of the $j^{\text {th }}$ and $k^{\text {th }}$ links last. The integral over the other intermediate points is facilitated by Eqs. (B10, B15) of Appendix B. The expression that results is a low-dimensional Gaussian integral over the end points of links $j$ and $k$ that can be explicitly computed. 
Proceeding in this manner we obtain

$$
\begin{aligned}
\delta \mathcal{K}_{B_{\perp}, B_{\|}}^{(n)}(\mathbf{r}, \mathbf{r}) & =\left(\frac{e B_{\|} \Delta}{\hbar}\right)^{2}\left[f_{1}+f_{2}-f_{3}\right] \\
f_{1}(n, \gamma, d) & \approx \frac{n \gamma}{\pi} \sum_{k=3}^{n}\{\operatorname{coth}[k \sqrt{\gamma}] \operatorname{coth}[(n-k) \sqrt{\gamma}]-1 \\
& \left.+\frac{d}{\sqrt{\gamma}} \operatorname{coth}[k \sqrt{\gamma}]+\frac{d}{\sqrt{\gamma}} \operatorname{coth}[(n-k) \sqrt{\gamma}]\right\} \\
& \times\{\sinh [k \sqrt{\gamma}] \sinh [(n-k) \sqrt{\gamma}]\}^{-1} \\
& \times\left(\operatorname{coth}[k \sqrt{\gamma}]+\operatorname{coth}[(n-k) \sqrt{\gamma}]+4 \frac{d}{\sqrt{\gamma}}\right)^{-2} \\
f_{2}(n, \gamma, d) & \approx \frac{2 n}{\pi} \frac{\sqrt{\gamma}}{\sinh [n \sqrt{\gamma}]}\{\sqrt{\gamma} \operatorname{coth}[n \sqrt{\gamma}]+d\} \\
f_{3}(n, \gamma, d) & \approx \frac{n}{\pi} \sqrt{\gamma} \frac{1}{\sinh [n \sqrt{\gamma}]}
\end{aligned}
$$

where $\gamma \equiv\left(B_{\perp} / 2 B_{e}\right)^{2}$ is a measure of the dynamical strength of the applied perpendicular field and we have taken $n \gg 1, \gamma \ll 1$ and $d \ll 1$. This is justified because weak-localization is dominated by long paths, the applied field is dynamically weak, and we are concerned with long-ranged roughness in this section. We have also taken $n \gamma \ll 1$, justifiable a posteriori, because we find $\delta \mathcal{K}^{(n)}$ decays exponentially for $n \gg 1 / \sqrt{\gamma}$.

Using Eq. (27) for the conductance and Eq. (75) for $\delta \mathcal{K}_{B_{\perp}, B_{\|}}^{(n)}$, after considerable simplification, we obtain a lengthy expression for the parallel field magnetoconductance,

$$
\delta g=-2 \frac{e^{2}}{\hbar}\left(\frac{B_{\|} \Delta L}{\phi_{\mathrm{sc}}}\right)^{2} u\left(\eta, B_{\phi} / B_{\perp}\right)
$$

Here $\eta \equiv \phi_{\mathrm{sc}} / 2 \pi B_{\perp} L^{2}$ and

$$
u\left(\eta, B_{\phi} / B_{\perp}\right)=\eta \int_{1}^{\infty} d y \frac{\ln y}{y^{2 B_{\phi} / B_{\perp}}}\left[v(y, \eta)-\frac{2}{y^{2}-1}\right],
$$

with

$$
\begin{aligned}
v(y, \eta) & =\frac{2}{\left(y^{2}-1\right)} \frac{\left[\left(1+2 \eta+2 \eta^{2}\right)\left(y^{2}-1\right)+4 \eta\right]}{\left[(1+2 \eta)^{2}\left(y^{2}-1\right)+8 \eta\right]} \\
& -\left(\frac{\eta}{f^{3 / 2}}\left[(1+2 \eta)\left(y^{2}-1\right)^{2}+(8+4 \eta)\left(y^{2}-1\right)+8\right]\right. \\
& \left.\times \ln \left\{\frac{\left[\sqrt{f}-2 \eta\left(y^{2}-1\right)\right]^{2}-\left(y^{2}-1\right)^{2}}{\left[\sqrt{f}+2 \eta\left(y^{2}-1\right)\right]^{2}-\left(y^{2}-1\right)^{2}}\right\}\right) ; \\
f(y, \eta) & =\left(y^{2}-1\right)\left[(1+2 \eta)^{2}\left(y^{2}-1\right)+8 \eta\right] .
\end{aligned}
$$

Eqs. (76)-(78) are the main results of this section. They give the shift in conductance from the standard weak-localization lineshape due to the applied in-plane field $B_{\|}$. Remarkably $\delta g$ is independent of $l_{e}$.
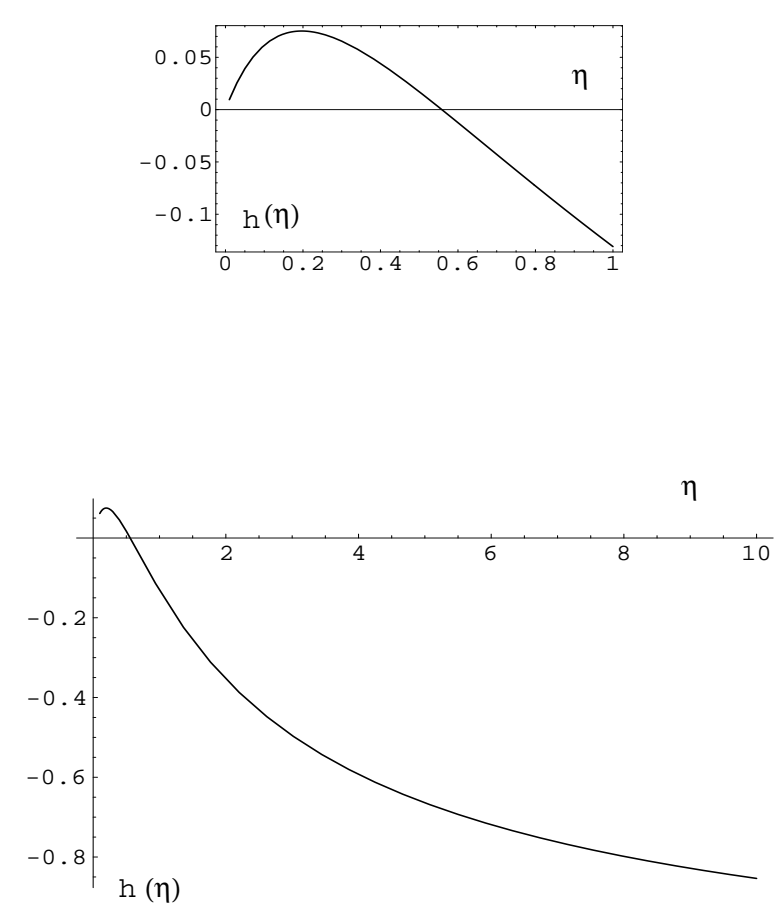

FIG. 5. Plot of $h(\eta) / \eta$ as a function of $\eta=\phi_{\mathrm{sc}} /\left(2 \pi L^{2} B_{\perp}\right)$. The inset shows the small $\eta$ behavior and the zero of $h(\eta)$ in detail. $h(\eta)$ describes the perpendicular field dependence of the in-plane magnetoresistance in the geometric limit, $l_{e} \ll L \ll l_{\phi}$; see Eq. (79).

Since the expression for $\delta g$ is complicated, it is instructive to examine several special cases. First, by taking the limit $B_{\perp} \rightarrow 0$ it is possible to recover the result of the previous subsection, Eq. (67). Next consider the strong perpendicular field limit $B_{\perp} l_{\phi}^{2} \gg \phi_{\mathrm{sc}}$. In this limit

$$
\delta g=-2 \frac{e^{2}}{\hbar}\left(\frac{B_{\|} \Delta L}{\phi_{\mathrm{sc}}}\right) h(\eta)
$$

with

$$
h(\eta)=\eta \int_{1}^{\infty} d y \ln y v(y, \eta)-\frac{\pi^{2}}{4} \eta
$$

A plot of $h(\eta)$ is shown in Fig. 5. It has the asymptotic behavior

$$
\begin{aligned}
h(\eta) & \approx \eta^{2} \text { for } \eta \ll 1 \\
& \approx-\frac{\pi^{2}}{8} \text { for } \eta \gg 1 .
\end{aligned}
$$

Note that $h(\eta)$ changes sign; it vanishes for $\eta \approx 0.558$.

Eq. (79) reveals that for $B_{\perp} l_{\phi}^{2} \gg \phi_{\mathrm{sc}}, \delta g$ depends only on the magnetic fields $B_{\|}$and $B_{\perp}$ and geometric parameters that characterize the rough interface, $\Delta$ and $L$. It is independent not only of $l_{e}$ but also of $l_{\phi}$. The intermediate regime in which $l_{e} \ll L$, but $L \ll l_{\phi}$, is particularly interesting. In this limit $B_{\phi} \ll \phi_{\text {sc }} / L^{2}$ and hence Eq. 
(79) applies in a range of $B_{\perp}$ over which $\eta$ varies from large to small. Thus the perpendicular field magnetoconductance curves at different values of $B_{\|}$cross at a point determined by the purely geometric condition

$$
B_{\perp} L^{2} \approx 1.79 \frac{h}{e} .
$$

For this reason we call this intermediate regime, $l_{e} \ll$ $L \ll l_{\phi}$, the geometric regime.

The opposite limit of extremely long-ranged roughness, $L \gg l_{\phi} \gg l_{e}$, we call the inhomogeneous broadening regime. In this limit, too, perpendicular magnetoconductance curves corresponding to different small $B_{\|}$ cross, but the crossing point is not determined by the purely geometric condition, Eq. (82). In this case the range of $B_{\perp}$ over which the simplified formula, Eq. (79), applies corresponds to very small $\eta$ and does not include the geometric crossing point, $\eta=0.558 \ldots$ We must therefore use the general expression Eq. (76) to determine the crossing point. Nonetheless in the inhomogeneous broadening regime the deviation from the conventional weak-localization lineshape and the existence of a crossing point has a simple interpretation. Since the random magnetic field is correlated over a length scale $L$, the sample breaks up into blocks of size $l_{\phi}$ each of which sees a slightly different (but uniform) perpendicular magnetic field, $B_{\perp}+\delta B$. For small $\delta B$, the shift in conductance of a particular block may be computed from the conventional weak-localization lineshape $g_{\mathrm{WL}}\left(B_{\perp}\right)$ by expanding in a Taylor series:

$$
\delta g \approx \frac{\partial}{\partial B_{\perp}} g_{\mathrm{WL}}\left(B_{\perp}\right) \delta B+\frac{1}{2} \frac{\partial^{2}}{\partial B_{\perp}^{2}} g_{\mathrm{WL}}\left(B_{\perp}\right) \delta B^{2}+\ldots
$$

Bearing in mind that the average value of the random field is zero and the typical value, $B_{\|} \Delta / L$, the average deviation is

$$
\delta g \sim B_{\|}^{2} \frac{\Delta^{2}}{L^{2}} \frac{\partial^{2}}{\partial B_{\perp}^{2}} g_{\mathrm{WL}}\left(B_{\perp}\right) .
$$

The curvature of $g_{\mathrm{WL}}\left(B_{\perp}\right)$ is positive at $B_{\perp}=0$. At large $B_{\perp} \gg \phi_{\mathrm{sc}} / l_{\phi}^{2}, g_{\mathrm{WL}}\left(B_{\perp}\right) \sim\left(e^{2} / h\right) \ln \left(B_{\perp} / B_{\phi}\right)$; hence the curvature is negative. Thus there must be an inflection point at which the curvature of $g_{\mathrm{WL}}\left(B_{\perp}\right)$ vanishes. According to Eq. (84) this is the crossing point. Substitution of the known asymptotic forms of $g_{\mathrm{WL}}\left(B_{\perp}\right)$ in Eq. (84) allows a check on the consistency of this interpretation. For small $B_{\perp}$ we recover the estimate given in the second line of Eq. (70). For $B_{\perp} \gg \phi_{\text {sc }} / l_{\phi}^{2}$ we obtain

$$
\delta g \sim \frac{e^{2}}{h} \frac{B_{\|}^{2} \Delta^{2}}{B_{\perp}^{2} L^{2}}
$$

in agreement with the small $\eta$ limit of Eq. (79).

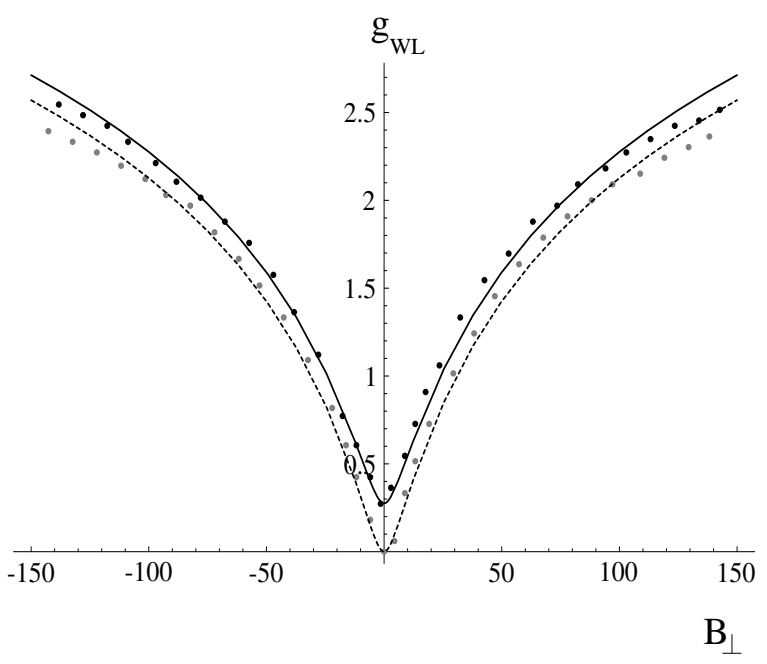

FIG. 6. Plot of the conductance (in units of $10^{-5}$ mhos) against perpendicular field $B_{\perp}$ (in Gauss), for the control device of Anderson et al. [6. Data points for $B_{\|}=0$ are grey; for $B_{\|}=1.5 \mathrm{~T}$, black. The dashed line is a fit to the weak-localization lineshape, from which Anderson et al. infer $l_{e}=0.085 \mu \mathrm{m}, l_{\phi}=0.76 \mu \mathrm{m}$. The solid curve shows the lineshape at $B_{\|}=1.5 \mathrm{~T}$ calculated from the expression for homogeneous broadening derived in this paper, Eqs. (44) and (55). All the parameters for this curve are independently determined. The values of $\Delta=1.35 \AA$ and $L=0.055 \mu \mathrm{m}$ are obtained from atomic force microscope images; for $l_{e}$ and $l_{\phi}$, we use the values cited above.

\section{COMPARISON TO EXPERIMENT AND RELATED WORK}

In their experiments Wheeler and collaborators apply a fixed $B_{\|}$and measure the conductance as a function of $B_{\perp}$. They have reported such lineshape measurements for many devices and have also explored the effect of varying electron density on the lineshape. The data of Anderson et al. [6] are particularly interesting because they have independently measured the interface roughness by etching away the oxide and imaged the exposed silicon surface using atomic force microscopy. Hence we focus on the measurements reported in that paper.

Fig. 6 shows the measured magnetoconductance of the control device at $B_{\|}=0$ (grey) and $B_{\|}=1.5 \mathrm{~T}$ (black). By fitting the $B_{\|}=0$ data to a weak-localization lineshape (dashed curve in Fig. 6), Eq. (44), Anderson et al. infer $l_{e}=0.085 \mu \mathrm{m}, l_{\phi}=0.76 \mu \mathrm{m}$. Atomic force microscope measurements yield $\Delta=1.35 \AA$ and $L=0.055 \mu \mathrm{m}$. Thus the device is in the short-range correlated regime. The black curve shows the calculated lineshape using the independently measured values of $\Delta, L, l_{e}$ and $l_{\phi}$. No parameters are adjusted except for a constant offset. The fit is good, comparable, for example, to the fit to weaklocalization lineshape at $B_{\|}=0$.

Anderson et al. also report measurements on a sec- 
ond device (called the textured device). This device is fabricated on a silicon wafer that has been roughened by argon sputtering prior to gate oxidation. As a result the textured device has atypical values of $\Delta$ and $L . L$ for the textured sample is comparable to $l_{e}$. Consistent with this we do not obtain a good zero-parameter fit to either the short or long-range roughness expressions derived here. Following Anderson et al. we can force-fit the data to the homogeneously broadened lineshape; but the value of $\Delta^{2} L$ we then obtain is inconsistent with the atomic force microscope images.

Finally it is interesting to compare the experiments of Wheeler et al. to other work on random or periodic magnetic fields. Such fields have been realized by gating a GaAs/AlGaAs heterostructure with type II superconducting film [1. 12; by randomly depositing type I superconducting grains [13] or ferromagnetic dysprosium dots 14 on the heterostructure surface; and by covering the device with a rough macroscopic magnet [15]. In work most closely related to Wheeler and co-workers, Gusev et al. have prepatterned the substrate by drilling a lattice of holes so that the interface at which the twodimensional electron gas forms is periodically modulated [16]. An inhomogeneous magnetic field leads to many interesting features in the magnetoresistance even in the classical and ballistic regimes; many experiments have focused on these effects. Except for the work of Rammer and Shelankov [17] noted below, much of the theoretical literature too explores these regimes or focuses on the fundamental question of whether all eigenstates are localized in a random magnetic field.

Two experiments study quantum corrections in the weak-localization limit: In the work of Bending, von Klitzing and Ploog [11] the inhomogeneous magnetic field is produced by an Abrikosov lattice of flux lines in the superconducting gate; hence it is periodic. However the magnetoresistance is not sensitive to the arrangement of the vortices since the dephasing length is shorter than the distances between the vortices. This circumstance was analyzed by Rammer and Shelankov [17]. In the experiment of Gusev et al. too the magnetic field is essentially periodic rather than random allowing them to analyze it via a chessboard model [16]. Thus in both these works the inhomogeneous magnetic field is rather different from the random Gaussian correlated field studied in the experiments of Wheeler and co-workers and analyzed here.

\section{SUMMARY AND CONCLUSION}

In this paper we have been concerned with the influence of interface roughness on the magnetoconductance of MOSFETs. Using a Born-Oppenheimer approximation we have shown that effectively electrons respond to an applied in-plane magnetic field as though it were a random perpendicular field. Technically, the random magnetic field appears as a Berry phase term in the Born-Oppenheimer effective Hamiltonian. Eq. (9) shows that the random magnetic field is the component of $B_{\|}$normal to the rough surface $\mathcal{Z}_{g}(x, y)$. The BornOppenheimer analysis also gives precise meaning to the surface $\mathcal{Z}_{g}(x, y)$ : it is the location of the centre of the local subband wavefunction.

The next step is to analyze the magnetoconductance. This is controlled by the familiar physics of weaklocalization: at zero field there is a contribution to the conductance due to constructive interference of closed electron paths and their time-reversed counterparts. This contribution is suppressed by application of a perpendicular magnetic field because the electron acquires an Aharonov-Bohm phase as it traverses a closed path. The analysis of this paper shows that an in-plane field too has an effect due to interface roughness. The electron acquires a Berry's phase equal to the flux of the random field through the closed path. The effect of the random Berry's phase on the conductance is the main focus of this paper.

To calculate the effect it is necessary to sum the standard divergent series of Cooperon diagrams (Fig. 3). We find it useful to reformulate this sum in a manner reminiscent of the central limit theorem. Feynman graphs of high order control the sum. In these graphs we are able, without significant error, to replace pairs of Green function lines with suitable Gaussian factors. At zero field, the $n^{\text {th }}$ order diagram is then essentially the weight of a closed $n$-step Gaussian random walk [Eq. (27)]. For the known case of an applied perpendicular field, this formulation brings out clearly the relationship between the classic weak-localization lineshape (Eq. 44) and the distribution of directed area for closed random walks (see Eqs. 38 and 39 and Appendix C). The method also proves useful when an in-plane field is applied for longrange correlated roughness $\left(L \gg l_{e}\right)$. In this case the magnetoconductance is related to the directed area distribution of an interacting random walk (see Eqs. 63 and 73). To make contact with experiment it is sufficient to analyze this problem perturbatively. We do not attempt a complete solution of the interacting random walk or calculation of the full magnetoconductance for long-range correlated roughness. These problems are left open for future work.

For short-range correlated roughness, however, we are able to obtain the full magnetoconductance. The dependence of the conductance on perpendicular field, $B_{\perp}$, still has the classic weak-localization lineshape (Eq. 44) when an in-plane field is applied, just as it does at $B_{\|}=0$. The only effect of the in-plane field is to enhance the dephasing rate (Eq. 52). In terms of field scales, $B_{\phi}$ becomes larger; the effective $B_{\phi}$ is given by Eq. (55). Hence we say that short-range correlated roughness produces homogeneous broadening of the weak-localization lineshape.

The departure from the conventional lineshape is more 
striking in case of long-range correlated roughness (although quantitatively smaller if all parameters except $L$ are held fixed). The change in conductance, $\delta g$, when a parallel field is applied ("parallel field magnetoconductance") is given by Eq. (76). The most remarkable feature of this expression is that it is independent of $l_{e} . \delta g$ is determined entirely by the magnetic field, geometric parameters of the interface, $\Delta$ and $L$, and the dephasing length, $l_{\phi}$. It may be of either sign (positive near $B_{\perp}=0$ and negative for large $B_{\perp}$ ). The expression for $\delta g$ is lengthy and cannot be more compactly expressed in terms of known special functions. It can be numerically computed and plotted with ease and precision; however it is worthwhile to examine several special cases.

In the limit of extremely long-ranged roughness, $L \gg$ $l_{\phi} \gg l_{e}$, the parallel field magnetoconductance has a simple interpretation: the sample breaks up into independent blocks of size $l_{\phi}^{2}$ each of which sees a slightly different magnetic field. Hence in this limit we say that the effect of an in-plane field and roughness is to inhomogeneously broaden the weak-localization line. Pursuing this interpretation we can understand the sign change in $\delta g$ and recover its form in various circumstances [see the discussion following Eqs. (70) and (82)].

The limit of intermediate-range correlated roughness, $l_{\phi} \gg L \gg l_{e}$, is particularly striking. For sufficiently large perpendicular field, $B_{\perp} l_{\phi}^{2} \gg \phi_{\mathrm{sc}}, \delta g$ becomes independent not only of $l_{e}$ but also $l_{\phi}$ [see Eq. (79)]. Essentially the parallel magnetoconductance is controlled by the function $h(\eta)$ where $\eta=\phi_{\mathrm{sc}} /\left(2 \pi L^{2} B_{\perp}\right)$ is a measure of the perpendicular flux (see Fig. 5).

Another special case, useful for making contact with experiment [7] is the parallel field magnetoconductance for $B_{\perp}=0$ (Eq. 67). In this case, $\delta g$, in units of $e^{2} / h$, is a product of the squared flux $\left(B_{\|} \Delta L\right)^{2}$, determined entirely by the field and the geometry of the interface, and the function $f(\alpha) . f(\alpha)$ describes the crossover as $l_{\phi}$ is varied relative to $L\left(\alpha=l_{\phi}^{2} / L^{2}\right.$. See Fig. 4$)$.

Wheeler and co-workers have shown experimentally in a number of cases that the effect of an in-plane field is to produce homogeneous broadening. Our analysis of the short-range correlated regime provides a theoretical justification of such fits to data and allows quantitative information about the interface roughness to be extracted. It is encouraging that for the control sample studied by Anderson et al. [6], for which the interface parameters are independently measured via atomic force microscopy, our formulae give a satisfactory zero parameter fit (Fig. 6 ). Unfortunately, our analysis shows that in the shortrange regime, magnetoresistance measurements will not separately yield $\Delta$ and $L$ : instead they provide the combination $\Delta^{2} L$. Thus it will be necessary to combine magnetoconductance measurements with other techniques to separately obtain $\Delta$ and $L$.

The roughness of the interface is controlled by its processing. For example, Anderson et al. [6] are able to increase both $\Delta$ and $L$ by argon sputtering the silicon surface prior to gate oxidation. It would be desirable to create devices with long-range correlated roughness in order to experimentally observe, for example, the geometric regime described above. At the same time, for long-range correlated roughness, the prospects are much better for extracting both $\Delta$ and $L$ from magnetoconductance measurements alone, provided the measurements can be made with sufficient precision. Eq. (76) provides the means to study the feasibility of such experiments.

Another potential application of our analysis is related to efforts to engineer the MOSFET interface to have specific structure, for example, a structure that is periodic in one direction like a corrugated sheet 29]. Weaklocalization magnetoconductance measurements would provide a non-destructive way to check that structures have been successfully fabricated. It should be a straightforward and interesting extension of our analysis to calculate the magnetoresistance signature of various simple periodic structures.

\section{ACKNOWLEDGEMENTS}

It is a pleasure to acknowledge many stimulating conversations with Albert Chang, Rachel Lombardi, Satya Majumdar, Don Monroe, and Bob Wheeler. H. Mathur is supported by NSF Grant DMR 98-04983 and an Alfred P Sloan Research fellowship and acknowledges the hospitality of the Aspen Center for Physics.

\section{APPENDIX A: ADIABATIC APPROXIMATION}

Here we briefly discuss the conditions for the validity of the Born-Oppenheimer approximation used in Section II. Roughly, we want only the low-lying states of the lowest subband to be occupied and we want the gap between subbands to be large. For this purpose, and to ensure the two dimensionality of the electron gas, we need the Fermi energy to be small compared to the subband spacing. The subband spacing is of order $\hbar^{2} / m \ell^{2}$ where $\ell$ is the length scale over which the electron is confined. Hence this condition may be phrased as

$$
\lambda_{f} \gg \ell \text {. }
$$

To obtain additional conditions we return to the Schrödinger Eq. (1) and seek a solution of the form

$$
\Psi(x, y, z)=\sum_{n} \psi_{n}(x, y) \phi_{n}(z ; x, y) .
$$

For simplicity we limit discussion to the case of zero magnetic field. $\psi_{n}(x, y)$ then obeys [9,21] 


$$
\begin{aligned}
& {\left[-\frac{\hbar^{2}}{2 m} \nabla^{2}+U_{\mathrm{imp}}(x, y)+E_{n}(x, y)+W_{n}(x, y)\right] \psi_{n}(x, y)} \\
& +\sum_{m \neq n} H_{n m} \psi_{m}(x, y)=E \psi_{n}(x, y) .
\end{aligned}
$$

Here

$$
H_{n m}=\frac{\hbar^{2}}{m}\langle n ; x, y|\nabla| m ; x, y\rangle . \nabla+\frac{\hbar^{2}}{2 m}\left\langle n ; x, y\left|\nabla^{2}\right| n ; x, y\right\rangle .
$$

Eq. (A4) constitutes an exact reformulation of the Schrödinger Eq. (1). The Born-Oppenheimer approximation consists of keeping only one term in the sum (A2) and hence omitting the off-diagonal terms in (A3).

In a commonly used model of the interface 20 it is assumed that the confining potential has the form

$$
U_{\text {conf }}(z ; x, y)=\mathcal{U}[z-\zeta(x, y)] .
$$

Here $\zeta(x, y)$ is the elevation of the silicon-oxide boundary. In this model the mean elevation of the local subband wavefunction exactly tracks the interface; hence the surface $\mathcal{Z}_{g}(x, y)$, probed by weak-localization magnetoresistance, has the same statistics as the silicon-oxide interface $\zeta(x, y)$, which is presumably imaged by atomic force microscopy. For a more general form of the confinement potential this precise correspondence between $\mathcal{Z}_{g}(x, y)$ and $\zeta(x, y)$ is lost but it seems reasonable that the two surfaces would have similar statistics, comparable correlation lengths and mean-square fluctuations.

Eq. (A5) implies that the local subband wave function is of the form

$$
\phi_{n}(z ; x, y)=\xi_{n}[z-\zeta(x, y)] .
$$

Using (A6) we can estimate the off-diagonal elements given by (A4). Requiring these to be small compared to subband spacing $\hbar^{2} / m \ell^{2}$, leads to

$$
\ell \nabla^{2} \mathcal{Z}_{g} \ll 1, \quad \nabla \mathcal{Z}_{g} \ll 1 .
$$

In summary, Eqs. (A1) and (A7) are the conditions for the validity of the Born-Oppenheimer approximation. Note that the same conditions can in fact be derived under less restrictive assumptions than Eq. (A5).

If we assume that $\mathcal{Z}_{g}$ is statistically the same as the surface imaged by atomic force microscopy, Eq. (A7) may be rewritten

$$
\frac{\ell \Delta_{\mathrm{AFM}}}{L^{2}} \ll 1, \quad \frac{\Delta_{\mathrm{AFM}}}{L} \ll 1 .
$$

For the control sample studied by Anderson et al. [6] $\Delta_{\mathrm{AFM}}=1.35 \AA, L=0.055 \mu \mathrm{m}, \lambda_{f}=100 \AA$; for the textured sample, $\Delta_{\mathrm{AFM}}=7.5 \AA, L=0.09 \mu \mathrm{m}, \lambda_{f}=$ $70 \AA$. Taking $\ell \approx 30 \AA$, it is easily seen that the BornOppenheimer approximation is applicable.

\section{APPENDIX B: COOPERON PATH INTEGRAL}

The purpose of this Appendix is to calculate the integral, Eq. (36). It is useful to first calculate

$$
\begin{aligned}
& D^{(n)}\left(y, y^{\prime}\right)=\int_{-\infty}^{+\infty} d y_{1} \ldots d y_{n} D^{(0)}\left(y, y_{1}\right) \ldots D^{(0)}\left(y_{n}, y^{\prime}\right) ; \\
& D^{(0)}\left(y, y^{\prime}\right)=\frac{1}{\sqrt{\pi}} e^{-\left(y-y^{\prime}\right)^{2}-g\left(y+y^{\prime}\right)^{2}} .
\end{aligned}
$$

It is easy to show by induction that $D^{(n)}$ is of the form

$$
D^{(n)}\left(y, y^{\prime}\right)=\frac{1}{\sqrt{\pi}} \frac{1}{A_{n}} e^{-a_{n}\left(y-y^{\prime}\right)^{2}-b_{n}\left(y+y^{\prime}\right)^{2}}
$$

with $a_{n} b_{n}=g$. By completing squares we find

$$
\begin{aligned}
D^{(n+1)}\left(y, y^{\prime}\right) & =\int_{-\infty}^{+\infty} d y_{1} D^{(0)}\left(y, y_{1}\right) D^{(n)}\left(y_{1}, y^{\prime}\right) \\
& =\frac{1}{\sqrt{\pi}} \frac{1}{A_{n+1}} e^{-a_{n+1}\left(y-y^{\prime}\right)^{2}-b_{n+1}\left(y+y^{\prime}\right)^{2}}
\end{aligned}
$$

provided $a_{n} b_{n}=g$. Here

$$
\begin{aligned}
\frac{1}{a_{n+1}} & =\frac{1}{a_{n}+g}+\frac{1}{b_{n}+1} ; \\
\frac{1}{b_{n+1}} & =\frac{1}{a_{n}+1}+\frac{1}{b_{n}+g} ; \\
A_{n+1} & =A_{n} \sqrt{a_{n}+b_{n}+1+g} .
\end{aligned}
$$

Using Eq. (B4) we can verify that $a_{n+1} b_{n+1}=g$ ensuring $D^{(n+2)}$ will also be of the form given in Eq. (B2). In principle the recurrence relations, Eq. (B4), determine $a_{n}, b_{n}$ and $A_{n}$; but in practice it is easier to follow a different method.

Experience with paths integrals suggests that the eigenfunctions of the kernel $D^{(0)}$ are $H_{m}(\sqrt{2 \alpha} y) e^{-\alpha y^{2}}$ with $\alpha$ suitably adjusted - of the same form as the eigenfunctions of a harmonic oscillator. To verify this conjecture and to fix $\alpha$ we use a generating function for the Hermite polynomials

$$
\exp \left(-x^{2}+2 x \sqrt{2 \alpha} y-\alpha y^{2}\right)=\sum_{m=0}^{\infty} \frac{x^{m}}{m !} H_{m}(\sqrt{2 \alpha} y) e^{-\alpha y^{2}} .
$$

Upon completing squares we find

$$
\begin{aligned}
& \int d y^{\prime} D^{(0)}\left(y, y^{\prime}\right) \exp \left(-x^{2}+2 x \sqrt{2 \alpha} y^{\prime}-\alpha y^{\prime 2}\right) \\
& =\frac{1}{1+\sqrt{g}} e^{\left[-\left(\frac{1-\sqrt{g}}{1+\sqrt{g}}\right)^{2} x^{2}+2\left(\frac{1-\sqrt{g}}{1+\sqrt{g}}\right) x 2 g^{1 / 4} y-2 \sqrt{g} y^{2}\right]} \\
& =\sum_{m=0}^{\infty} \frac{(1-\sqrt{g})^{m}}{(1+\sqrt{g})^{m+1}} \frac{x^{m}}{m !} H_{m}\left(2 g^{1 / 4} y\right) \exp \left(-2 \sqrt{g} y^{2}\right)
\end{aligned}
$$


provided $\alpha=2 \sqrt{g}$. This condition is imposed by requiring that the coefficient of the $y^{2}$ term in the second line of Eq. (B6) should be $\alpha$. Comparing Eqs. (B5) and (B6) we conclude that $H_{m}\left(2 g^{1 / 4} y\right) e^{-2 \sqrt{g} y^{2}}$ are eigenfunctions of $D^{(0)}$ with eigenvalue $(1-\sqrt{g})^{m} /(1+\sqrt{g})^{m+1}$ for $m=0,1,2, \ldots$.

Now let us evaluate

$$
\begin{aligned}
& \int_{-\infty}^{+\infty} d y^{\prime} D^{(n)}\left(y, y^{\prime}\right) \exp \left(-2 \sqrt{g} y^{\prime 2}-x^{2}+4 g^{1 / 4} x y\right) \\
& =\frac{1}{A_{n}} \frac{\sqrt{\pi a_{n}}}{a_{n}+\sqrt{g}} e^{\left[-2 \sqrt{g} y^{2}-\left(\frac{a_{n}-\sqrt{g}}{a_{n}+\sqrt{g}}\right)^{2} x^{2}+4 g^{1 / 4}\left(\frac{a_{n}-\sqrt{g}}{a_{n}+\sqrt{g}}\right) x y\right]} \\
& =\sum_{m=0}^{\infty} \frac{1}{m !} \frac{\sqrt{\pi a_{n}}}{A_{n}} \frac{\left(a_{n}-\sqrt{g}\right)^{m}}{\left(a_{n}+\sqrt{g}\right)^{m+1}} x^{m} H_{m}\left(2 g^{1 / 4} y\right) e^{-2 \sqrt{g} y^{2}} \\
& =\sum_{m=0}^{\infty} \frac{1}{m !} \frac{(1-\sqrt{g})^{m(n+1)}}{(1+\sqrt{g})^{(m+1)(n+1)}} x^{m} H_{m}\left(2 g^{1 / 4} y\right) e^{-2 \sqrt{g} y^{2}} .
\end{aligned}
$$

The integral in the first line of Eq. (B7) has been analyzed in two ways. First we use the ansatz for $D^{(n)}$, Eq. (B2), perform the integral by completing squares, and expand the result using the generating formula for the Hermite polynomials, Eq. (B5). The results are given in the second and third lines. Alternatively, we expand the exponential in the first line in terms of the eigenfunctions of $D^{(0)}$ using the generating formula, Eq. (B5), and note that the eigenfunctions of $D^{(0)}$ are also eigenfunctions of $D^{(n)}$ but with the eigenvalue raised to the $(n+1)^{\text {st }}$ power (considered as an integral operator, $D^{(n)}$ amounts to $n+1$ repeated applications of $D^{(0)}$ ). The result is given in the fourth line. Comparison of the third and fourth lines reveals

$$
\begin{aligned}
a_{n}= & \sqrt{g}\left[\frac{(1+\sqrt{g})^{n+1}+(1-\sqrt{g})^{n+1}}{(1+\sqrt{g})^{n+1}-(1-\sqrt{g})^{n+1}}\right] \\
b_{n}= & \sqrt{g}\left[\frac{(1+\sqrt{g})^{n+1}-(1-\sqrt{g})^{n+1}}{(1+\sqrt{g})^{n+1}+(1-\sqrt{g})^{n+1}}\right] \\
A_{n}= & \frac{\sqrt{\pi}}{2 g^{1 / 4}}\left[(1+\sqrt{g})^{n+1}+(1-\sqrt{g})^{n+1}\right]^{1 / 2} \\
& \times\left[(1+\sqrt{g})^{n+1}-(1-\sqrt{g})^{n+1}\right]^{1 / 2} .
\end{aligned}
$$

These expressions are the solution to the recurrence relation in Eq. (B4) with the initial condition $a_{0}=1, b_{0}=$ $g, A_{0}=1$.

In summary the integral $D^{(n)}$, defined in Eq. (B1) is given by Eqs. (B2) and (B8).

Now we evaluate Eq. (36) for $K_{B_{\perp}}^{(n)}\left(\mathbf{r}, \mathbf{r}^{\prime}\right)$. Rescaling the co-ordinates we obtain

$$
K_{B_{\perp}}^{(n)}\left(\mathbf{r}, \mathbf{r}^{\prime}\right)=\frac{1}{2 l_{e}^{2}} E^{(n)}\left(\mathbf{r}, \mathbf{r}^{\prime}\right)
$$

with

$$
\begin{aligned}
& E^{(n)}\left(\mathbf{r}, \mathbf{r}^{\prime}\right)=\int d \mathbf{r}_{1} \ldots d \mathbf{r}_{n} E^{(0)}\left(\mathbf{r}, \mathbf{r}_{1}\right) \ldots E^{(0)}\left(\mathbf{r}_{n}, \mathbf{r}^{\prime}\right) \\
& E^{(0)}\left(\mathbf{r}, \mathbf{r}^{\prime}\right)=\frac{1}{\pi} \exp \left[-\left|\mathbf{r}-\mathbf{r}^{\prime}\right|^{2}-i \beta\left(x-x^{\prime}\right)\left(y+y^{\prime}\right)\right]
\end{aligned}
$$

and $\beta=B_{\perp} / B_{e}$. In the Landau gauge $E^{(0)}$ depends only on $\left(x-x^{\prime}\right)$ and has the Fourier transform

$$
\begin{aligned}
& \frac{1}{\sqrt{\pi}} \exp \left[-\left(x-x^{\prime}\right)^{2}-i \beta\left(y+y^{\prime}\right)\left(x-x^{\prime}\right)\right] \\
& =\int_{-\infty}^{+\infty} \frac{d k}{2 \pi} \exp \left(-\frac{\left[k+\beta\left(y+y^{\prime}\right)\right]^{2}}{4}\right) e^{i k\left(x-x^{\prime}\right)} .
\end{aligned}
$$

By virtue of translational invariance

$$
\begin{aligned}
E^{(n)}\left(\mathbf{r}, \mathbf{r}^{\prime}\right)= & \int_{-\infty}^{+\infty} \frac{d k}{2 \pi} \int_{-\infty}^{+\infty} d y_{1} \ldots d y_{n} \frac{1}{\sqrt{\pi}^{n+1}} e^{i k\left(x-x^{\prime}\right)} \\
& \times e^{-\left[k+\beta\left(y+y_{1}\right)\right]^{2} / 4} \ldots e^{-\left[k+\beta\left(y_{n}+y^{\prime}\right)\right]^{2} / 4} \\
& \times e^{-\left(y-y_{1}\right)^{2}} \ldots e^{-\left(y_{n}-y^{\prime}\right)^{2}} .
\end{aligned}
$$

If we shift $y_{i} \rightarrow y_{i}-k / 2 \beta$

$$
\begin{aligned}
E^{(n)}\left(\mathbf{r}, \mathbf{r}^{\prime}\right)= & \int_{-\infty}^{+\infty} \frac{d k}{2 \pi} \int_{-\infty}^{+\infty} d y_{1} \ldots d y_{n} \frac{1}{\sqrt{\pi}^{n+1}} e^{i k\left(x-x^{\prime}\right)} \\
& \times \exp \left[-\frac{\beta^{2}}{4}\left(\bar{y}+y_{1}\right)^{2}+\ldots-\frac{\beta^{2}}{4}\left(y_{n}+\bar{y}^{\prime}\right)^{2}\right] \\
& \times e^{-\left(\bar{y}-y_{1}\right)^{2}+\ldots-\left(y_{n}-\bar{y}^{\prime}\right)^{2}}
\end{aligned}
$$

Here $\bar{y}=y-k / 2 \beta, \bar{y}^{\prime}=y^{\prime}-k / 2 \beta$ and the $k$-integral must be performed after the $y$-integrals. Evidently

$$
E^{(n)}\left(\mathbf{r}, \mathbf{r}^{\prime}\right)=\int_{-\infty}^{+\infty} \frac{d k}{2 \pi} e^{i k\left(x-x^{\prime}\right)} D^{(n)}\left(y-\frac{k}{2 \beta}, y^{\prime}-\frac{k}{2 \beta}\right) .
$$

$D^{(n)}$ is given by Eqs. (B2) and (B8) with $g \rightarrow \beta^{2} / 4$. Performing the remaining Gaussian integral over $k$ yields

$$
\begin{aligned}
E^{(n)}\left(\mathbf{r}, \mathbf{r}^{\prime}\right) & =\frac{1}{\pi q_{n}} e^{-a_{n}\left|\mathbf{r}-\mathbf{r}^{\prime}\right|^{2}-i \beta\left(y+y^{\prime}\right)\left(x-x^{\prime}\right)} \\
a_{n} & =\frac{[1+(\beta / 2)]^{n+1}+[1-(\beta / 2)]^{n+1}}{[1+(\beta / 2)]^{n+1}-[1-(\beta / 2)]^{n+1}} \\
q_{n} & =\frac{1}{\beta}\left[\left(1+\frac{\beta}{2}\right)^{n+1}-\left(1-\frac{\beta}{2}\right)^{n+1}\right] .
\end{aligned}
$$

Eqs. (B9), (B10) and (B15) constitute the final expression for $K_{B_{\perp}}^{(n)}\left(\mathbf{r}, \mathbf{r}^{\prime}\right)$.

The calculation of $K_{B_{\perp}}^{(n)}$ is similar to the solution of Schrödinger's equation for an electron in a magnetic field because the slowly varying eigenfunctions of $K_{B_{\perp}}^{(0)}$ obey the differential Eq. (33). However the derivation of Eq. (B15) goes beyond solution of the differential equation because we also obtain the short distance behavior of $K_{B}^{(n)}$.

Setting $\mathbf{r}=\mathbf{r}^{\prime}$ in Eq. (B15) we obtain

$$
\begin{aligned}
K_{B_{\perp}}^{(n-1)}\left(\mathbf{r}, \mathbf{r}^{\prime}\right) & =\frac{1}{2 \pi l_{e}^{2}} \frac{B_{\perp}}{B_{e}}\left[\left(1+\frac{B_{\perp}}{2 B_{e}}\right)^{n}-\left(1-\frac{B_{\perp}}{2 B_{e}}\right)^{n}\right]^{-1} \\
& \approx \frac{B_{\perp}}{2 \phi_{\mathrm{sc}}}\left[\sinh \left(\frac{n B_{\perp}}{2 B_{e}}\right)\right]^{-1}
\end{aligned}
$$


provided that $B_{\perp} / B_{e}$ is sufficiently small that not only is $B_{\perp} / B_{e} \ll 1$ but also $\left(B_{\perp} / B_{e}\right)^{2} n \ll 1$. The approximate expression in Eq. (B16) is the same as Eq. (41). For a given large $B_{e} / B_{\perp}$ Eq. (41) is accurate only for $n \ll$ $\left(B_{e} / B_{\perp}\right)^{2}$; but since $K_{B_{\perp}}^{(n)}\left(\mathbf{r}, \mathbf{r}^{\prime}\right)$ is exponentially small for $n \gg B_{e} / B_{\perp}$, no significant error is made by taking Eq. (41) to apply for all $n$.

\section{APPENDIX C: DIRECTED AREA DISTRIBUTION}

First let us make precise the meaning of directed area. Imagine an $n$-sided polygon drawn on a plane. The polygon is endowed with an orientation by assigning each edge a direction such that each vertex has one incoming edge and one outgoing. Roughly, the two orientations of a given polygon correspond to the two senses in which its perimeter may be circumnavigated.

Now suppose a magnetic field is applied perpendicular to the plane. Roughly, the directed area is the quantity by which the magnetic field must be multiplied in order to obtain the flux through the oriented polygon (in magnitude and sign). For a simple polygon with no selfintersection the directed area is equal in magnitude to the area; the sign is positive or negative depending on whether the orientation of the polygon is anti-clockwise or clockwise viewed from the direction in which the magnetic field points. For a polygon with self-intersection, the directed area is obtained by weighting the area of each infinitesimal area element by its winding number before adding them. Here the winding number of an area element is computed by drawing a vector from the element to the boundary of the polygon and counting the number of anticlockwise turns made by the vector as its tip circumnavigates the perimeter (clockwise turns count as negative turns). For a polygon with successive vertices at $\mathbf{r}, \mathbf{r}_{1}, \ldots, \mathbf{r}_{n-1}, \mathbf{r}$ immersed in a magnetic field pointing in the $z$-direction, the directed area is given by

$$
\begin{aligned}
a= & -\frac{1}{2}\left(x_{1}-x\right)\left(y_{1}+y\right)-\frac{1}{2}\left(x_{2}-x_{1}\right)\left(y_{2}+y_{1}\right)+\ldots \\
& \ldots-\frac{1}{2}\left(x-x_{n}\right)\left(y+y_{n}\right) .
\end{aligned}
$$

Let the probability density of drawing an $n$-sided oriented polygon with successive vertices at $\mathbf{r}, \mathbf{r}_{1}, \ldots, \mathbf{r}_{n-1}, \mathbf{r}$ be $\left(n / \pi^{n-1}\right) \exp \left[-\left|\mathbf{r}-\mathbf{r}_{1}\right|^{2}+\ldots+\left|\mathbf{r}_{n-1}-\mathbf{r}\right|^{2}\right]$. Eqs. (C1) and (B10) show that

$$
E^{(n-1)}(\mathbf{r}, \mathbf{r})=\frac{1}{n \pi} \int_{-\infty}^{+\infty} d a P(a) \exp (i 2 \beta a)
$$

Here $P(a)=$ probability distribution of the directed area for the Gaussian polygon ensemble defined above. By inverting the Fourier transform in Eq. (C2) and making use of Eq. (B15) for $E^{(n-1)}\left(\mathbf{r}, \mathbf{r}^{\prime}\right)$ we obtain

$$
\begin{aligned}
P(a) & =\int_{-\infty}^{+\infty} \frac{d \alpha}{2 \pi}\left(\frac{n \alpha}{2}\right)\left[\left(1+\frac{\alpha}{4}\right)^{n}-\left(1-\frac{\alpha}{4}\right)^{n}\right]^{-1} e^{-i \alpha a} \\
& \approx \int_{-\infty}^{+\infty} \frac{d \alpha}{2 \pi}\left(\frac{n \alpha}{4}\right)\left[\sinh \left(\frac{n \alpha}{4}\right)\right]^{-1} e^{-i \alpha a} \\
& =\frac{\pi}{n} \operatorname{sech}^{2}\left(\frac{2 \pi a}{n}\right) .
\end{aligned}
$$

The approximate form of the integrand in the second line above is valid only for $\alpha \ll 1 / \sqrt{n}$; but it may be extended to all $\alpha$ since the integrand is already exponentially small for $\alpha \gg 1 / n$.

Eq. (C3) is the formula for the directed area distribution. It coincides with Eq. (41) if we take the step size of the random walker to be $\sqrt{2} l_{e}$ instead of 1 as we have done in this Appendix.

The distribution of directed area for Brownian motion was analysed by Lévy [30] and restudied more explicitly in connection with electron transport by Argaman et al. [31]. Here however we require the area distribution $P_{n}(a)$ for a random walk with a finite number of steps $n$. The large $n$ behaviour of $P_{n}(a)$ was recently obtained by Bellissard et al. for random polygons on a square lattice by an interesting application of non-commutative geometry [32]. Their result agrees with the large $n$ limit of eq (C3).

[1] P.A. Lee and T.V. Ramakrishnan, Rev. Mod. Phys. 57, 287 (1985).

[2] J. Rammer, Quantum Transport Theory (Perseus Books, Reading MA, 1998).

[3] S. Hikami, A. Larkin and Nagaoka, Prog. Theor. Phys. 63, 707 (1980); B.L. Altshuler, D.E. Khmel'nitskii, A.I. Larkin and P.A. Lee, Phys. Rev. B 22, 5142 (1980).

[4] For a review see G. Bergmann, Phys. Rep. 101, 1 (1984).

[5] P.M. Menz, R.G. Wheeler, C.T. Foxon and J.J. Harris, Appl. Phys. Lett. 50, 603 (1987); P.M. Mensz and R.G. Wheeler, Phys. Rev. B35, 2844 (1987).

[6] W.R. Anderson, D.R. Lombardi, R.G. Wheeler and T.-P. Ma, IEEE Electron Dev. Lett. 14, 351 (1993).

[7] Similar experiments have been performed by A. M. Chang (private communication).

[8] M.V. Berry, Proc. Roy. Soc. (Lond) A 292, 45 (1984).

[9] A. Shapere and F. Wilczek Geometric Phases in Physics (World Scientific, Singapore, 1989).

[10] D. Loss, P.M. Goldbart and A.V. Balatsky, Phys. Rev. Lett. 65, 1655 (1990); A. Stern, Phys. Rev. Lett. 68, 1022 (1992); S.A. van Langen, H.P.A. Knops, J.C.J. Paaschens and C.W.J. Beenakker, Phys. Rev. B 59, 2102 (1999).

[11] S.J. Bending, K. von Klitzing, and K. Ploog, Phys. Rev. Lett. 65, 1060 (1990); Phys. Rev. B 42, 9859 (1990).

[12] A. Geim, S. Bending and I. Grigorieva, Phys. Rev. Lett. 69, 2252 (1992); Phys. Rev. B 49, 5749 (1994).

[13] A. Smith, R. Taboryski, L.T. Hansen, C.B Sørensen, P. 
Hedegård and P.E. Lindelof, Phys. Rev. B 50, 14726 (1994).

[14] P.D. Ye, D. Weiss, K. von Klitzing, K. Eberl and H. Nickel, App. Phys. Lett. 67, 1441 (1995); Phys. Rev. Lett. 74, 3013 (1995); P.D. Ye, D. Weiss, G. Lütjering, R.R. Gerhardts, K. von Klitzing, K. Eberl, H. Nickel and G. Weimann, in The Physics of Semiconductors, M. Scheffler and R. Zimmermann (eds) (World Scientific, 1996).

[15] F.B. Mancoff, R.M. Clarke, C.M. Marcus, S.C. Zhang, K. Campman and A.C. Gossard, Phys. Rev. B 51, 13269 (1995);

[16] G.M. Gusev, U. Gennser, X. Kleber, D.K. Maude, J.C. Portal, D.I. Lubyshev, P. Basmaji, M. de P.A. Silva, J.C. Rossi and Yu. V. Nastaushev, Phys. Rev. B 53, 13641 (1996);

[17] J. Rammer and A.L. Shelankov, Phys. Rev. B 36, 3135 (1987); B.L. Altshuler and L.B. Ioffe, Phys. Rev. Lett. 69, 2979 (1992); S.C. Zhang and D.P. Arovas, Phys. Rev. Lett. 72, 1886 (1994); A.G. Aronov, A.D. Mirlin and P. Wölfle, Phys. Rev. B 49, 16609 (1994); D.K.K. Lee and J.T. Chalker, Phys. Rev. Lett. 72, 1510 (1994); V.I. Fal'ko, Phys. Rev. B 50, 17406 (1994).

[18] G. Timp, R.E. Howard, and P.M. Mankiewich, in Nanotechnology, edited by G. Timp (Springer-Verlag, New York, 1999) pp. 7-87 and references therein.

[19] D. Monroe, private communication.

[20] For a thorough discussion see T. Ando, A.B. Fowler and F. Stern, Rev. Mod. Phys. 54, 437 (1982).

[21] For a modern textbook discussion see R. Shankar, Principles of Quantum Mechanics (Plenum, $2^{\text {nd }}$ edition, 1994) Chapter 21.

[22] The general principles of linear response theory are discussed in many textbooks. See for example, A. Fetter and F. Walecka, Quantum Theory of Many Particle Systems (McGraw-Hill, New York, 1971). For a linear response analysis of non-interacting electrons in a magnetic field see H.U. Baranger and A.D. Stone, Phys. Rev. B 40, 8169 (1989).

[23] Impurity averaged perturbation theory is discussed by A.A. Abrikosov, L.P. Gorkov and I.E. Dzyaloshinskii, Methods of Quantum Field Theory in Statistical Physics (Dover, NY, 1975) Section 39.2.

[24] For a microscopic derivation see I.L. Aleiner, B.L. Altshuler and M.E. Gershenson, Phys. Rev. Lett. 82, 3190 (1999); cond-mat/9808053; and references therein.

[25] S. Chakravarty and A. Schmid, Phys. Rep. 140, 193 (1986).

[26] A.J. McKane and M. Stone, Annals of Phys. 131, 36 (1981).

[27] P.M. Morse and H. Feshbach, Methods of Theoretical Physics, vol 1, p 422-423 (McGraw-Hill, New York 1953).

[28] S.F. Edwards and M. Doi, Introduction to Polymer Dynamics, (Oxford Univ Press, 1986).

[29] E. Kapon in "Epitaxial Microstructures, Semiconductors and Semimetals", A.C. Gossard (ed), vol 40, p259 (Academic, NY 1994); Sakaki (private communication).

[30] P. Lévy, Processus Stochastiques et Mouvement Brownien (Gauthier-Villars, Paris, 1948).

[31] N. Argaman, Y. Imry and U. Smilansky, Phys Rev B47, 4440 (1993).
[32] J. Bellissard, C.J. Camacho, A. Barelli and F. Claro, J. Phys A: Math. Gen, 30, L707 (1997). 